\title{
Potent but transient immunosuppression of T-cells is a general feature of erythroid progenitor cells
}

Tomasz M. Grzywa1 ${ }^{1,2,3}$, Anna Sosnowska ${ }^{1,4}$, Zuzanna Rydzynska1 ${ }^{1}$, Michal Lazniewski ${ }^{5,6}$, Dariusz Plewczynski ${ }^{5,7}$, Klaudia Klicka ${ }^{2,8}$, Milena Malecka ${ }^{9}$, Anna Rodziewicz-Lurzynska ${ }^{10}$, Olga Ciepiela9 ${ }^{9}$, Magdalena Justyniarska ${ }^{1}$, Paulina

Pomper $^{11}$, Marcin M. Grzybowski ${ }^{11}$, Roman Blaszczyk ${ }^{11}$, Michal Wegrzynowicz ${ }^{12}$, Agnieszka Tomaszewska ${ }^{13}$, Grzegorz Basak ${ }^{13}$, Jakub Golab ${ }^{1,14^{*}}$, Dominika Nowis ${ }^{1,3^{*}}$

${ }^{1}$ Department of Immunology, Medical University of Warsaw, Warsaw, Poland ${ }^{2}$ Doctoral School of the Medical University of Warsaw, Warsaw, Poland

${ }^{3}$ Laboratory of Experimental Medicine, Medical University of Warsaw, Warsaw, Poland

${ }^{4}$ Postgraduate School of Molecular Medicine, Medical University of Warsaw, Warsaw, Poland

${ }^{5}$ Laboratory of Functional and Structural Genomics, Centre of New Technologies, University of Warsaw, Warsaw, Poland

${ }^{6}$ Centre for Advanced Materials and Technologies, Warsaw University of

Technology, Warsaw, Poland

${ }^{7}$ Faculty of Mathematics and Information Science, Warsaw University of Technology, Warsaw, Poland

${ }^{8}$ Department of Methodology, Medical University of Warsaw, Warsaw, Poland

${ }^{9}$ Department of Laboratory Medicine, Medical University of Warsaw, Warsaw, Poland

${ }^{10}$ Central Laboratory, University Clinical Center of Medical University of Warsaw,

Warsaw, Poland

${ }^{11}$ OncoArendi Therapeutics, Warsaw, Poland

${ }^{12}$ Laboratory of Molecular Basis of Neurodegeneration, Mossakowski Medical

Research Institute, Polish Academy of Sciences, Warsaw, Poland

${ }^{13}$ Department of Hematology, Transplantation and Internal Medicine, Medical

University of Warsaw, Warsaw, Poland

${ }^{14}$ Centre of Preclinical Research, Medical University of Warsaw, Warsaw, Poland

Keywords: erythroid progenitor cells, anemia, arginase, reactive oxygen species, immunosuppression, phenylhydrazine

Running title: EPCs and immunosuppression

${ }^{*}$ corresponding authors:

Jakub Golab, M.D., Ph.D.

e-mail: jakub.golab@wum.edu.pl

Dominika Nowis, M.D., Ph.D.

e-mail: dominika.nowis@wum.edu.pl

Department of Immunology

Medical University of Warsaw

5 Nielubowicza Str.

02-097 Warsaw, Poland

Phone: +48-5992199 


\section{Abstract}

Erythroid progenitor cells (EPCs) have been recently recognized as potent immunoregulatory cells with defined roles in fetomaternal tolerance and immune response to infectious agents in neonates and cancer patients. Here, we show that

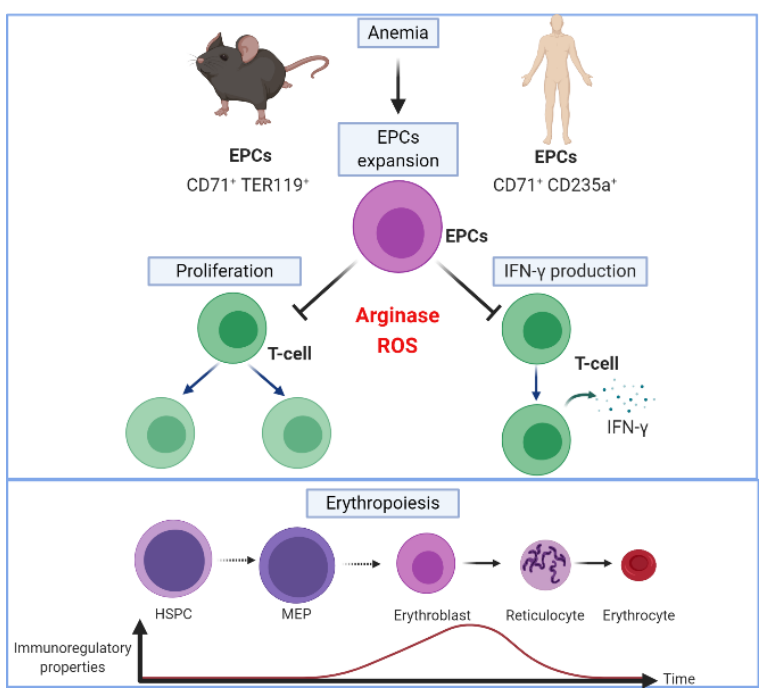
early-stage EPCs are enriched in anemia, have high levels of arginase 2 (ARG2) and reactive oxygen species (ROS). EPCs expansion in anemic mice leads to the Larginine depletion in the spleen microenvironment resulting in the suppression of Tcell responses. In humans with anemia, EPCs expand and express both ARG1 and ARG2 that participate in suppressing the proliferation and production of IFN-y from Tcells. EPCs differentiated from peripheral blood mononuclear cells potently suppress T-cell proliferation and this effect is the most prominent for CD49d ${ }^{\text {hi }}$ CD71 hi EPCs. The suppressive properties disappear during erythroid differentiation as more differentiated EPCs as well as mature erythrocytes lack significant immunoregulatory properties. Our studies provide a novel insight into the role of EPCs in the regulation of immune response. 


\section{Introduction}

Erythroid progenitor cells (EPCs) normally reside in the bone marrow and are precursors to over $2 \times 10^{11}$ of oxygen-transporting red blood cells (RBCs) generated per day ${ }^{1}$. When steady-state erythropoiesis becomes insufficient to meet increased tissue oxygen demands, EPCs are released from the bone marrow to the circulation and expand in the extramedullary hematopoietic sites. Recent studies revealed an unexpected complexity of EPCs functions in the human body. EPCs arose as a relevant population of cells regulating immunity $2,3,4$. Initially, EPCs were reported to suppress both innate and humoral immune response in neonates ${ }^{4,5,6}$ and it was suggested that their immunomodulatory functions are restricted to early life events ${ }^{4}$. However, further studies revealed a crucial role of EPCs in the regulation of multiple phenomena such as fetomaternal tolerance ${ }^{7}$, immune response in cancer patients ${ }^{8,9}$, systemic inflammation in colitis ${ }^{10}$, and anti-viral response in human immunodeficiency virus (HIV) infection ${ }^{11}$, as well as SARS-CoV-2-induced disease (COVID-19) ${ }^{12}$. It has been reported that CD45+ EPCs induced by advanced tumors inhibit CD8 ${ }^{+}$and CD4+ T-cell proliferation and impair antimicrobial immunity ${ }^{9}$. Interestingly, the authors demonstrated that EPCs from mice with acute hemolytic anemia, induced by systemic phenylhydrazine $(\mathrm{PHZ})$ administration, are not immunosuppressive as compared with EPCs from tumor-bearing mice ${ }^{9}$. This could lead to the conclusion that only EPCs in new-borns and patients with advanced cancer have robust immunosuppressive properties. In this study, we provide evidence that EPCs in anemic mice do have immunoregulatory properties, but $\mathrm{PHZ}$ used to induce hemolysis affects the mechanisms of immune suppression used by these cells masking their phenotype. Moreover, we comprehensively elucidate the role of EPCs in the regulation of immune response in both mice and humans and demonstrate that 
bioRxiv preprint doi: https://doi.org/10.1101/2021.01.18.427109; this version posted January 19, 2021. The copyright holder for this preprint (which was not certified by peer review) is the author/funder, who has granted bioRxiv a license to display the preprint in perpetuity. It is made available under aCC-BY-NC-ND 4.0 International license.

93 immunomodulatory properties of EPCs are robust but transient and disappear during

94 their maturation. 


\section{Results}

\section{EPCs expand in the spleens of anemic mice}

We initially compared the expansion of EPCs in 3 days old neonatal and adult anemic mice (Fig. 1a). Non-hemolytic anemia (NHA) was induced by phlebotomy and hemolytic anemia $(\mathrm{HA})$ was induced either by administration of $\mathrm{PHZ}(\mathrm{HA}-\mathrm{PHZ})$ or anti-TER119 antibodies (HA-TER119) (see Supplementary Fig. 1 for hematological parameters of these mice). EPCs expanded in the spleens of anemic mice as compared with controls, but were significantly less frequent than in neonatal mice (Fig. 1b). However, EPCs numbers in the spleen were substantially higher in anemic mice than in neonates or controls (Fig. 1c). The percentage of EPCs increased also in the blood of anemic mice (Supplementary Fig. 2b), but remained unchanged in the bone marrow (Supplementary Fig. 2a). Some studies indicated that EPCs at the earliest stages of differentiation express CD45 and have the most potent immunomodulatory properties ${ }^{8,9}$. The proportion of $\mathrm{CD}^{2} 5^{+}$to $\mathrm{CD} 45^{-}$EPCs was the highest in HA-PHZ mice and the lowest in neonatal mice (Fig. 1d). Analysis of developmental stages of EPCs based on cell size and CD44 levels (Fig. 1e $)^{13}$ revealed enrichment of less differentiated EPCs in anemic mice compared to nonanemic controls (Fig. 1f, Supplementary Fig. 2c).

\section{T-cell immune response is impaired in anemic mice}

Next, we sought to determine whether anemia might impair the function of the immune system. To this end we assessed selected functionalities of myeloid, B- and T-cells in control and anemic mice. In contrast to neonatal mice ${ }^{4,5}$, production of 
TNF- $\alpha$ by splenic CD11 $b^{+}$cells after stimulation with heat-killed E. coli (HKEc) (Supplementary Fig. 3a) or the concentration of anti-ovalbumin (OVA) IgG antibodies after OVA-ALUM immunization (Supplementary Fig. 3b,c) was unimpaired in adult anemic mice as compared with healthy controls. Intriguingly, we found that the proliferation of adoptively transferred SIINFEKL-specific OT-I T-cells in response to OVA stimulation was decreased in the spleen of NHA mice compared to healthy controls (Fig. 2a,b). Since in the spleen of anemic mice the expansion of EPCs was the most substantial (Supplementary Fig. 3d), we hypothesized that EPCs might be responsible for T-cells suppression. Indeed, EPCs isolated from the spleens of both $\mathrm{HA}$ and NHA anemic mice (Fig. 2c) suppressed proliferation of $\mathrm{CD} 4^{+}{ }^{+}$-cells that were activated with anti-CD3/CD28 beads (Fig. 2d). Altogether, these data document a rather selective impairment of T-cells response by EPCs in anemic mice.

\section{Murine EPCs have high ROS level and express ARG2}

Both ROS generation and expression of L-Arg-degrading enzyme arginase were previously identified as the effectors of the immunoregulatory activity of neonatal $\mathrm{EPCs}^{4}, 14$. Accordingly, we found that both cytoplasmic and nuclear ROS levels were higher in anemia-induced EPCs as compared with RBCs (Fig. 3a, Supplementary Fig. 4a,b) and they reached the highest values in the EPCs at the earliest stages of their maturation (Supplementary Fig. 4c,d). Interestingly, in contrast to human $E P^{2} s^{12}$, ROS levels in murine EPCs were significantly lower than in the cells of nonerythroid lineages such as myeloid -cells and T-cells (Fig. 3b).

Murine EPCs expressed mitochondrial isoform of arginase, ARG2 (Fig. 3c), but had almost undetectable cytosolic ARG1 (Fig. 3d). Similar to ROS, the levels of ARG1 
and ARG2 were the highest in early-stage EPCs and consequently decreased during maturation (Supplementary Fig. 5a,b). Intriguingly, while the percentage of $A R G 2^{+}$ EPCs was similar in all groups (Fig. 3c), the fraction of $A R G 1^{+}$cells was substantially higher in HA-PHZ mice as determined by intracellular staining (Fig. 3d). This finding seems counterintuitive considering that ARG-dependent degradation of L-arginine leads to T-cell suppression ${ }^{15},{ }^{16}$, and EPCs from HA-PHZ mice exerted the weakest suppressive effects on T-cells proliferation. Increased expression of ARG1 in HAPHZ EPCs was further confirmed by ARG1 mRNA detection (Supplementary Fig. 6a) and in reporter B6.129S4-Arg1tm1Lky/J mice that express YFP under Arg1 promoter (Fig. 3e,f) indicating that flow cytometry findings were not artifactual. HA-PHZ EPCs had increased expression of ARG2 mRNA as compared with NHA EPCs (Supplementary Fig. 6b), but no increase in ARG2 protein levels was observed (Supplementary Fig. 6c). Surprisingly, despite robust upregulation of ARG1 levels, total arginase activity in both EPCs isolated from HA-PHZ mice and EPCsconditioned medium was lower even than that in EPCs from NHA mice (Fig. 3g,h). Moreover, EPCs cultured ex vivo in the presence of PHZ strongly upregulated ARG1 expression (Fig. 3i).

\section{PHZ targets arginase and suppresses its activity}

Increased expression with a concomitant decrease in arginase activity suggested an interaction between $\mathrm{PHZ}$ and arginase. Further studies showed that indeed $\mathrm{PHZ}$ inhibits the activity of recombinant human ARG1 and ARG2, with an $\mathrm{IC}_{50}$ of $1017 \mu \mathrm{M}$ and $61 \mu \mathrm{M}$, respectively (Fig. 4a). However, $\mathrm{PHZ}$ did not affect the production of nitric oxide (NO) by nitric oxide synthase, which is also using L-arginine as a substrate (Fig. 4b). To elucidate how PHZ interacts with ARG1 and ARG2 a molecular docking 
simulation was carried out with PHZ, L-arginine as well as 2-amino-6-borono-2-(2(piperidin-1-yl)ethyl)hexanoic acid (ABH) that is a strong ARG1 inhibitor ${ }^{17}$. PHZ binds to the active sites of all arginases, where it forms several polar interactions involving D128, D232, or T246 (Supplementary Fig. 7a). Thus, it may block the entry of other molecules to the active site. However, predicted binding energies suggest that among the tested ligands PHZ has the weakest affinity for arginases, and thus a significant concentration of this compound may be required to induce any biological effect, which indeed is the case in vivo. The transient nature of interactions between $\mathrm{PHZ}$ and arginases was also confirmed by a short 100 ns MD simulation (Supplementary Fig. 7b,c). The ligand remained bound to the active site for only 15$30 \%$ of the simulation time, despite its initial placement inside the ligand-binding pocket. The analysis of electrostatic surface potential revealed the presence of a large, negatively charged area around the substrate-binding pocket of ARG1 that likely plays a role in attracting positively charged L-arginine to the catalytic site (Fig. 4c). Since $\mathrm{PHZ}$ in the presence of oxygen leads to the formation of free radicals and hydrogen peroxide ${ }^{18}$, we hypothesized that decreased ARG activity in EPCs from $\mathrm{HA}-\mathrm{PHZ}$ mice might emerge due to non-specific non-covalent interactions of $\mathrm{PHZ}$ with the catalytic pocket of ARG1 that leads to oxidative changes in the enzyme, decreased activity, and subsequent degradation. Indeed, incubation of recombinant ARG1 with $\mathrm{PHZ}$ in the presence of oxygen led to a significant increase in the carbonylation of the enzyme that was reduced by concomitant incubation with $\mathrm{N}$ acetylcysteine (ROSi) (Fig. 4d).

\section{EPCs degrade L-Arg and produce ROS leading to the suppression of T-cells}


Due to the interaction between $\mathrm{PHZ}$ and arginases, we chose NHA as a model of anemia-induced EPCs for further studies. We found that CD4+ T-cells stimulated with anti-CD3/CD28 beads in the presence of EPCs showed downregulation of activation markers CD25 and CD69, which was less pronounced for CD62L (Fig. 5a). Both arginase inhibitor (ARGi, OAT-1746 ${ }^{19}$ ) and ROS inhibitor (ROSi, N-acetylcysteine) nearly completely restored the proliferation of T-cells that was inhibited by co-culture with EPCs isolated from NHA mice (Fig. 5b), similar to EPCs isolated from neonates (Supplementary Fig. 8). Likewise, EPCs-conditioned medium had a suppressive effect on T-cell proliferation, and supplementation with either of L-arginine or ARGi restored T-cell proliferation to percentages akin to the control group (Fig. 5c).

To further study the role of ARG2 in the modulation of immune response by EPCs, we assessed the suppressive effects of EPCs isolated from anemic mice lacking functional $\operatorname{Arg} 2$ gene $\left(\operatorname{Arg} 2^{-/}, A r g 2^{\text {m1 } 1 \text { Weo/J }}\right.$ mice $\left.^{20}\right) . A r g 2^{--}$mice had a slightly increased percentage of $\mathrm{ARG} 1^{+}$EPCs compared to wild type mice $\left(\operatorname{Arg} 2^{+/+}\right)$ (Supplementary Fig. 9a), however, with no significant changes in total ARG1 level in EPCs (Supplementary Fig. 9b). EPCs from Arg2 $^{-/-}$mice had substantially diminished suppressive effects on T-cell proliferation compared to $\operatorname{Arg}^{+/+}$EPCs (Fig. 5d), which confirmed the critical role of ARG2 in the regulation of T-cells function by murine EPCs.

Further studies revealed that expansion of ARG-expressing EPCs in the anemic mice led to the substantial increase of the arginase activity (Fig. 5e) caused by increased ARG2 but not ARG1 levels in the spleen (Fig. 5f-h). Even though the concentration of L-arginine was only slightly decreased in the serum of anemic mice (Supplementary

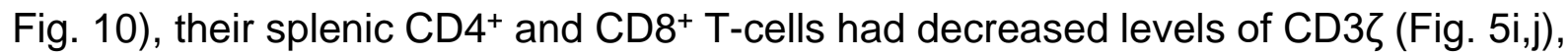


EPCs results in the depletion of L-arginine in the microenvironment leading to the Tcells impairment. Consequently, ex vivo stimulation of T-cells with anti-CD3/CD28 beads in the presence of EPCs resulted in a decrease of $\mathrm{CD} 3 \zeta$, which was diminished by ARGi and completely restored by the combination of ARGi and ROSi (Fig. 5k,I). Altogether, these results show that EPCs suppress T-cells response in anemic mice via both arginase and ROS.

\section{EPCs expand in the blood of anemic individuals and suppress T-cells}

Then, we sought to investigate the role of EPCs in anemic patients (Supplementary Table 1, Supplementary Table 2). The percentage of EPCs $\left(C D 71{ }^{+} \mathrm{CD} 235 \mathrm{a}^{+}\right)$in peripheral blood was substantially increased in anemic individuals (Fig. 6a,b). The number of EPCs in the blood (Fig. 6c) reversely correlated with the hemoglobin concentration (Fig. 6d) and was the highest in patients with moderate and severe anemia (Fig. 6e).

In anemic patients EPCs constituted a substantial fraction of peripheral blood mononuclear cells (PBMCs) (Fig. 6f,g) and were predominantly at the latest stages of differentiation with a very small percentage of CD45+ EPCs (Supplementary Fig. S11a). We found that the production of IFN-y in response to CD3/CD28 stimulation was suppressed in T-cells from anemic individuals when compared to non-anemic controls (Fig. 6h). However, T-cells proliferation was unimpaired in anemic patients (Supplementary Fig. S11b,c). Moreover, there were no differences in the production of TNF- $\alpha$ by myeloid cells between anemic and control individuals in response to killed bacteria (Supplementary Fig. S11d). 


\section{EPCs from human bone marrow suppress T-cells proliferation}

Human bone marrow is predominantly composed of mature erythrocytes, however, EPCs constituted a substantial cell population (Fig. 7a). EPCs in the bone marrow are at the earliest stages of differentiation (Supplementary Fig. 12a) and are predominantly $\mathrm{CD} 45^{+}$(Supplementary Fig. 12b). Similar to murine EPCs, their counterparts in the human bone marrow express ARG2 (Fig. 7b). Importantly, human erythroid cells also express ARG1 (Fig. 7c). EPCs from human bone marrow suppressed proliferation of both $\mathrm{CD} 4^{+}$and $\mathrm{CD} 8^{+} \mathrm{T}$-cells (Fig. $7 \mathrm{~d}, \mathrm{e}$ ). This effect was diminished by theARGi, which confirmed arginase-dependent effect.

\section{Suppression of T-cells function is a general feature of erythroid cells which} disappears during their maturation

Our results suggest that T-cells suppression is a common feature of both murine and human EPCs. Further studies confirmed that similarly to EPCs, model human erythroleukemic cell lines, K562, HEL92.1.7, and TF-I (Supplementary Fig. 13a) have substantial arginase activity (Supplementary Fig. 13b), express both ARG1 (Supplementary Fig. 13c) and ARG2 (Supplementary Fig. 13d), and have high ROS levels (Supplementary Fig. 13e). Erythroid cell lines potently suppressed both CD4+ and $\mathrm{CD}^{+}$human $\mathrm{T}$-cell proliferation (Fig. 8a,b). However, induction of erythroid differentiation of K562 cells (K562-E) by sodium butyrate 22 (Supplementary Fig. 14a) resulted in decreased suppressive effects on T-cell proliferation (Supplementary Fig. 14b). K562-E cells had decreased ARG2 but not ARG1 levels (Supplementary Fig. 14c) and decreased total arginase activity as compared with non-differentiated K562 cells (Supplementary Fig. 14d). Downregulation of ARG2 was most probably caused 
by mitophagy, a crucial process during erythroid differentiation ${ }^{23}$, as evidenced by decreased signal from mitochondrial probe in differentiated K562-E cells (Supplementary Fig. 14e). These observations strongly suggest that the immunoregulatory properties of EPCs may diminish during maturation.

Therefore, we next sought to establish a model of ex vivo differentiation of erythroid cells. To this end EPCs were expanded and differentiated from PBMC of healthy markers, including CD71, CD235a, CD36, and CD49d, and had high expression of CD44 and CD45 (Supplementary Fig. 15b). Similar to their bone marrow counterparts, EPCs expanded from PBMCs had high levels of both ARG1 and ARG2 (Supplementary Fig. 16a,b) and potently suppressed both $\mathrm{CD}^{+}$and CD8+ human Tcell proliferation (Fig. 8d,e).

Next, we aimed to study possible changes in immunoregulatory properties of erythroid cells during differentiation into RBC. First, we investigated whether hematopoietic stem and progenitor cells (HSPCs) exert immunosuppressive effects. Mobilized hematopoietic stem cells obtained from peripheral blood (peripheral blood stem cells, PBSCs, Supplementary Fig. 17a) had high ARG1 as well as ARG2 levels (Supplementary Fig. 17b) and included only a small percentage of EPCs (Supplementary Fig. 17c). Despite high arginase expression, PBSCs had no impact on T-cell proliferation (Supplementary Fig. 17d,e).

Then, we demonstrated that EPCs differentiated from PBMCs (Fig. 8f) exert robust, but transient suppressive properties, that disappear during erythroid differentiation

(Fig. 8g-i). We found that of all EPCs developmental stages CD45+CD44+CD49d ${ }^{\text {hi }}$ EPCs most strongly inhibited T-cells proliferation. Loss of suppressive properties 
290 latter being a marker of the transition to the reticulocyte stage 24,25 . Subsequent EPCs differentiation resulted in a complete loss of suppressive effects on T-cells. Similarly, mature erythrocytes obtained from healthy donors had no impact on T-cell proliferation (Supplementary Fig. 18). Altogether, we show that human EPCs possess robust but transient suppressive properties that disappear during maturation.

\section{Discussion}

In this study, we demonstrate that suppression of T-cells is a general feature of murine and human EPCs that expand during anemia. Anemic EPCs via arginases and ROS suppress proliferation and production of IFN-y by T-cells. Using continuous human erythroid cell culture, we show that the immunoregulatory properties of EPCs are transient and disappear during maturation.

Recent studies expanded our understanding of the many roles played by EPCs expanded by different triggers ${ }^{3}$. Immunoregulatory functions of EPCs were reported for the first time in neonates that are characterized by a physiological abundance of EPCs ${ }^{4}$. Neonatal EPCs suppress anti-bacterial immunity via ARG2 by decreasing the production of proinflammatory cytokines by myeloid cells ${ }^{4}$ and by suppressing antibody production in response to $B$. pertussis ${ }^{5}$. We found that in adult mice anemia induced the expansion of early-stage EPCs that had the highest expression of ARG2. Even though, neither ARG2-expressing EPCs nor recombinant ARG1 did not suppress the production of TNF-a from myeloid cells. However, 311 arginases seem to primarily impair T-cells by decreasing their activation and proliferation $^{26}$. Accordingly, we observed decreased proliferation of adoptively transferred OT-I cells in the spleen of anemic mice, which was reflected ex vivo in the 
co-culture of murine T-cells with EPCs. Expansion of EPCs in the spleen of anemic mice resulted in the increased ARG activity in the spleen leading to the L-arginine starvation of T-cells, decreased levels of $\mathrm{CD} 3 \zeta$, and suppressed proliferation. Moreover, human EPCs expressed both ARG1 and ARG2 and suppressed T-cell proliferation in an ARG-dependent manner. Thus, expansion of ARG-expressing EPCs in anemia may induce immune suppression, similar to the expansion of ARGexpressing myeloid cells in cancer ${ }^{27}$ and during pregnancy ${ }^{28}$.

EPCs were also reported to modulate immune response via ROS in tumorbearing mice and cancer patients ${ }^{9}$. We found that ROSi restored T-cell proliferation in co-culture with EPCs from anemic mice to a similar extend as ARGi. ROS also may decrease $\mathrm{CD} 3 \zeta$ in T-cells ${ }^{29}$. However, ROSi restored CD3 $\zeta$ decreased by EPCs only in combination with ARGi, which confirms that ARG cooperates with ROS in EPCs to induce T-cells hyporesponsiveness to proliferative triggers.

Importantly, we demonstrated that previously described lack of immunosuppressive capacities of EPCs in anemic mice ${ }^{9}$ resulted from the interaction between PHZ used to induced anemia and ARGs. PHZ-induced hemolytic anemia is one of the most commonly used models of anemia. PHZ leads to the formation of ferrihemoglobin from oxyhemoglobin and production of free radicals that disrupt the interactions between hem and globin chains leading to the formation of Heinz bodies and hemolysis ${ }^{18}$. However, PHZ-induced EPCs are less effective in suppressing Tcell proliferation as compared with EPCs isolated from neonatal or other anemic mice. We show that $\mathrm{PHZ}$ targets $\mathrm{ARGs}$, critical immunomodulating enzymes, in an oxidation-dependent mechanism, reminiscent of Heinz bodies formation. It needs to be considered in future studies that the interaction between $\mathrm{PHZ}$ and ARGs may have considerable effects on the obtained results. 
We further demonstrated that EPCs expand in anemic patients and suppress T-cells. Anemia correlates with worse outcomes in many diseases, including pneumonia $^{30}$ or cancer $^{31}$. Moreover, preoperative anemia is associated with an increased risk of infection and mortality in patients undergoing surgery ${ }^{32}, 33$. In line with our results, a recent study showed that anemia status influences the blood

344 transcriptome with enrichment of erythrocyte differentiation genes as well as ARG1 in anemic children, but decreased signatures of $\mathrm{CD}^{+}$T-cell activation and 346 differentiation ${ }^{34}$. It remains unknown to which extent EPCs are responsible for 347 immune suppression and whether in these conditions supplementation of iron, 348 vitamin $\mathrm{B} 12$, or administration of erythropoiesis-stimulating agents including EPO 349 may restore immune response. blood cells from a hematopoietic stem cell (HSC) and is strictly regulated by multiple factors $^{35}$. Recent studies demonstrated that immunomodulatory properties are strong in early-stage CD45+ EPCs in contrast to more mature CD45- EPCs $^{8}, 9,14,36$. We showed that human EPCs acquire immunomodulatory properties during erythroid differentiation and are the most potent in CD71 hiCD49d ${ }^{\text {hiCD }} 44{ }^{\text {hi }}$ CD45+ EPCs. Further erythroid maturation is associated with the disappearance of the suppressive 357 properties.

The exact role of transient immunomodulatory properties of EPCs remains elusive. It was suggested that expansion of EPCs in neonates provides tolerance to harmless antigens, including the commensal microbiota ${ }^{4}$, and minimalize damage caused by inflammation in the intestines ${ }^{4}$, liver ${ }^{6}$, and lungs ${ }^{37}$ during first days of 362 postnatal life. In adults, the role of EPCs seems to be similar. Recent studies demonstrated that stress erythropoiesis is a key inflammatory response ${ }^{38}$, therefore, 
expansion of EPCs may suppress chronic inflammation. Indeed, transfer of EPCs suppressed inflammatory response and attenuated the wasting syndrome in murine models of colitis ${ }^{10}$. In cancer, which is characterized by a chronic inflammation ${ }^{39}$, EPCs substantially expand and suppress immune response facilitating tumor growth 368 and increasing the susceptibility to pathogens ${ }^{9}$. On the other side, impaired 369 immunoregulatory properties of EPCs may exacerbate damage caused by

370 inflammation ${ }^{40}$. Moreover, EPCs by suppressing production of IFN- $\gamma$, a crucial

371 inflammatory cytokine and a potent inhibitor of erythropoiesis ${ }^{41,42}$, may allow 372 maintaining erythropoiesis. underlying suppressed cell-mediated immunity and anti-bactericidal capacity of

375 leucocytes ${ }^{43}$ and the impaired of T-cell mediated immunity in anemic children ${ }^{44}$. 


\section{Methods}

377

Reagents. Recombinant human ARG1 was obtained from Biolegend (San Diego, CA, USA), recombinant murine Arg1 was obtained from Cloud-Clone Corp., arginase inhibitor OAT-1746 was synthesized at OncoArendi Therapeutics, Warsaw, Poland. All other reagents, if not otherwise stated, were obtained from Sigma-Aldrich.

\section{Cell lines}

K562, HEL92.1.7 and TF-I cell lines were purchased from American Type Culture Collection (ATCC). Cells were cultured in RPMI-1640 medium supplemented with 10\% heat-inactived fetal bovine serum (FBS, HyClone), 2 mM L-glutamine (SigmaAldrich) $100 \mathrm{U} / \mathrm{ml}$ penicillin and $100 \mu \mathrm{g} / \mathrm{ml}$ streptomycin (Sigma-Aldrich) at $37^{\circ} \mathrm{C}$ in an atmosphere of $5 \% \mathrm{CO}_{2}$ in the air. Additionally, TF-I cells medium contained $2 \mathrm{ng} / \mathrm{ml}$ recombinant human GM-CSF (R\&D Systems). Cells have been cultured for no longer than 4 weeks after thawing and were regularly tested for Mycoplasma contamination using PCR technique and were confirmed to be negative.

\section{Human samples}

Peripheral blood samples were obtained from patients hospitalized in the Central Teaching Clinical Hospital, Medical University of Warsaw or treated in the Outpatient Clinic of Central Teaching Clinical Hospital, Medical University of Warsaw, Warsaw. The study was conducted in accordance with the Declaration of Helsinki. Study was approved by the Bioethical Committee of Medical University of Warsaw (KB/8/2021). Patients with or without anemia based on WHO diagnostic criteria ${ }^{45}$ were enrolled to the study. Patients with proliferative diseases, including cancer, were excluded from the study. The blood samples were obtained by venipuncture and subjected to complete blood count evaluation. The remaining blood was used for further examination. Flow cytometry was performed as described below. CountBright ${ }^{\mathrm{TM}}$ 
Absolute Counting Beads (ThermoFisherScientific) were used for EPCs counting. Peripheral blood mononuclear cells (PBMC) were purified from whole blood of anemic and non-anemic patients by density separation using Lymphoprep (STEMCELL Technologies).

Human bone marrow aspirates from healthy donors were commercially obtained from Lonza. Bone marrow donors were both males $(n=6)$ and females $(n=3)$ at the age of 23-45. Mobilized peripheral blood stem cells (PBSCs) were obtained from familial donors from the material remaining after allogeneic stem cell transplantation. Informed consent was obtained from the PBSC cell donors.

Human T-cells were isolated from PBMC obtained by Histopaque-1077 (Sigma Aldrich) or Lymphoprep (STEMCELL Technologies) separation from buffy coats from healthy volunteers, commercially obtained from the Regional Blood Centre in Warsaw, Poland.

\section{Anemia animal models}

C57BL/ 6 both male and female 8 to 14 -week-old mice were obtained from the Animal House of the Polish Academy of Sciences, Medical Research Institute (Warsaw,

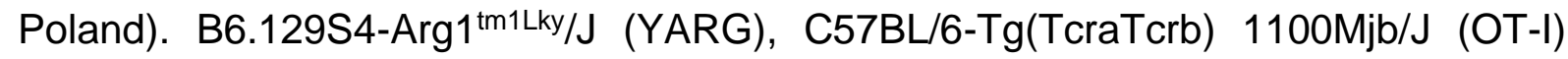
and $\mathrm{Arg} 2^{\mathrm{tm} 1 \text { Weo/J }}$ (Arg2 functional knockout, $\mathrm{Arg}^{-1-}$ ) mice were purchased from the Jackson Laboratories. The experiments were performed in accordance with the guidelines approved by the II Local Ethics Committee in Warsaw (approval No. WAW2/117/2019 and WAW2/143/2020) and in accordance with the requirements of EU (Directive 2010/63/EU) and Polish (Dz. U. poz. 266/15.01.2015) legislation. To induce non-hemolytic anemia (NHA) mice were phlebotomized 4 and 2 days before harvest. At least $100 \mu \mathrm{l}$ of blood was collected each time. To induce hemolytic anemia (HA), mice were injected intraperitoneally (i.p.) three days before harvest with 
$50 \mathrm{mg}$ per kg body weight of phenylhydrazine ( $\mathrm{PHZ}$ ) hydrochloride solution (HA-PHZ) or mice were injected intravenously (i.v.) six days before harvest with $45 \mu \mathrm{g}$ of antiTER119 monoclonal antibody (TER-119, BioXCell) into caudal vein. Blood was obtained from facial veins as a terminal procedure and examined using Sysmex XN2000 Hematology Analyzer. The parameters of complete blood counts and reference intervals $^{46}$ are presented in Supplementary Fig. 1. Plasma L-arg concentration was determined with ultra-performance liquid chromatography tandem mass spectrometry (UPLC-MS/MS) method on Waters Xevo TQ-S mass spectrometer equipped with Waters Acquity UPLC chromatograph (Waters) in the Mass Spectrometry Lab at the Institute of Biochemistry and Biophysics, Polish Academy of Sciences, Warsaw, Poland.

\section{Antibodies}

Fluorophore- or biotin-conjugated antibodies specific for mouse cell-surface antigens and cytokines were as follows: anti-CD71 (8D3, NovusBio; R17217, eBioscience), anti-TER119 (TER-119, BioLegend), anti-CD45.2 (104, BD Biosciences), anti-CD44 (IM7, BioLegend), anti-CD3e (145-2C11, eBioscience), anti-CD4 (GK1.5, eBioscience; RM4-5. eBioscience), anti-CD8a (53-6.7, eBioscience), anti-CD69 (H1.2F4, eBioscience), anti-CD25 (PC61.5, eBioscience), anti-CD62L (MEL-14, Invitrogen), anti-CD3 zeta (H146-968, Abcam), anti-IFN-y (XMG1.2, eBioscience), anti-TNF- $\alpha$ (MP6-XT22, eBioscience), anti-Arg1 (polyclonal, IC5868P/F, R\&D Systems), anti-Arg2 (ab81505, Abcam), goat anti-rabbit lgG (Invitrogen).

Fluorophore- or biotin-conjugated antibodies specific for human cell-surface antigens and cytokines were as follows: anti-CD71 (CY1G4, BioLegend, DF1513, NovusBio), anti-CD235a (HI264, BioLegend), anti-CD44 (IM7, BioLegend), anti-CD45 (HI30, BD Bioscience), anti-CD49d (9F10, eBioscience), anti-CD36 (NL07, eBioscience), anti- 
451 CD34 (561, BioLegend), anti-CD3 (OKT3, eBioscience), anti-CD4 (RPA-T4,

452 eBioscience), anti-CD8a (RPA-T8, eBioscience), anti-IFN-Y (4S.B3, BioLegend), anti-

453 TNF- $\alpha$ (MAb11, BD Bioscience), anti-Arg1 (polyclonal, IC5868P/F, R\&D Systems),

454 anti-Arg2 (ab137069, Abcam), goat anti-rabbit lgG (Invitrogen).

\section{Flow cytometry analysis}

456 Flow cytometry was performed on FACSCanto II (BD Biosciences) or Fortessa X20

457 (BD Biosciences) operated by FACSDiva software. For data analysis Flow Jo v10.6.1

458

459

460

461

462

463

464

465

466

467

468

469

470

471

472

473

474

475 software (TreeStar) or BD FACSDiva software (BD Biosciences) were used. Fluorochrome-conjugated antibodies used for the staining are listed above. For cell surface staining, cells were stained with Zombie $\mathrm{NIR}^{\mathrm{TM}}$, Zombie UV ${ }^{\mathrm{TM}}$ or Zombie Aqua $^{\mathrm{TM}}$ Fixable Viability Kit (BioLegend), blocked on ice with $5 \%$ normal rat serum in FACS buffer (PBS; $1 \%$ BSA, $0.01 \%$ sodium azide) and then incubated for $30 \mathrm{~min}$ on ice with fluorochrome-labelled antibodies. After washing in FACS buffer, cells were immediately analyzed. For intracellular staining, membrane-stained cells were fixed using Fixation Buffer for $30 \mathrm{~min}$, followed by a wash with permeabilization buffer, and staining with an antibody diluted in permeabilization buffer for 30 min (Intracellular

Fixation \& Permeabilization Buffer Set, eBioscience). For anti-Arg2 indirect intracellular staining, cells were fixed using Fixation Buffer for $30 \mathrm{~min}$, followed by a wash with permeabilization buffer, and staining with anti-Arg2 antibody for $1 \mathrm{~h}$, followed by a wash with permeabilization buffer and staining with fluorochromeconjugated goat anti-rabbit IgG for $30 \mathrm{~min}$. Gating strategies used to analyze the flow cytometry data are presented in Supplementary Figures 19-36.

\section{IFN-Y and TNF- $\alpha$ production assay}

Murine splenocytes were isolated from anemic or healthy mice and human PBMC were isolated from the blood of anemic or non-anemic patients. Cells were plated in 
476 round-bottomed 96 -well plates $\left(1 \times 10^{6}\right.$ cell per well) in L-arginine-free RPMI-medium

477 (SILAC RPMI-medium, Thermofisher Scientific) supplemented with $10 \%$ dialyzed

478 FBS (Thermofisher Scientific), $2 \mathrm{mM}$ glutamine, $100 \mathrm{U} / \mathrm{ml}$ penicillin, $100 \mu \mathrm{g} / \mathrm{ml}$ were stimulated with Heat Killed E. coli 0111:B4 (HKEc, InvivoGen) at the concentration $1 \times 10^{6}$ cells per $\mathrm{ml}$ or Dynabeads T-Activator CD3/CD28 (ratio 1:2, Thermofisher Scientific) for $6 \mathrm{~h}$ for murine cells or $12 \mathrm{~h}$ for human cells in the presence with cell surface antigens-binding antibodies, followed by fixation, permeabilization and intracellular staining for IFN- $\gamma$ and TNF- $\alpha$. Flow cytometry was performed on Fortessa X20 (BD Biosciences).

\section{In vivo OVA immunization and analysis of humoral response}

488

489

490

491

492

493

494

495

496

497

498

499

Control and NHA mice was immunized with albumin from chicken egg white (OVA, Ovalbumin) from Sigma (Grade VII). Each mouse received $25 \mu \mathrm{g}$ of OVA with Imject ${ }^{\mathrm{TM}}$ Alum Adjuvant (ALUM, Thermofisher Scientific) at ratio $1: 1$ in the final volume $100 \mu \mathrm{l}$ per mouse administered i.p. After 14 days, mice were challenged once again with the same dose of OVA-ALUM. NHA mice were divided into three groups. NHA before mice were phlebotomized before first immunization, NHA boost mice were phlebotomized before second OVA immunization, and NHA both were phlebotomized before first and second immunization (see Supplementary Fig. 3b). Untreated mice received Imject ${ }^{\mathrm{TM}}$ Alum Adjuvant without OVA. Blood was obtained from mice 14 days after second immunization, plasma was isolated and stored at $80^{\circ} \mathrm{C}$. Concentration of anti-OVA IgG antibodies was determined using AntiOvalbumin IgG1 (mouse) ELISA Kit (Cayman Chemical).

\section{In vivo proliferation assay}


501 OVA (SIINFEKL)-specific $\mathrm{CD}^{+} \mathrm{T}$ cells were isolated from the spleen and lymph nodes of OT-I mice, labelled with CTV (as described below) and transferred into the caudal tail vein of host C57BL/6 mice at a cell number of $7 \times 10^{6}$ in $150 \mu$ of PBS.

504

Twenty-four hours post OT-I T-cells inoculation, host mice were challenged with 7.5 $\mu \mathrm{g}$ of full-length OVA protein (grade $\mathrm{V}$, Sigma Aldrich) injected into the caudal tail vein. Three mice from controls were injected only with PBS (negative control). On day 3 post OVA immunization, splenocytes were harvested, stained with OVAspecific MHC tetramers (iTAg Tetramer/PE-H-2 K ${ }^{\mathrm{b}}$ OVA (SIINFEKL), MBL Inc., WA, USA) to detect OT-I CD8 ${ }^{+}$T-cells, followed by anti-CD3 and anti-CD8 staining, and analyzed for proliferation by flow cytometry. The gate for proliferating cells (CTVlow) was set using unstimulated negative control.

\section{T-cell proliferation assay}

Murine T-cells were isolated from spleens of healthy 6 -week old C57BL/6 mice using EasySep ${ }^{\mathrm{TM}}$ Mouse $\mathrm{CD}^{+}$or $\mathrm{CD}^{+} \mathrm{T}$-Cell Isolation Kit (STEMCELL Technologies) according to the manufacturer's protocols. Human T-cells were isolated from peripheral blood mononuclear cells (PBMC) isolated from buffy coats commercially obtained from the Regional Blood Centre in Warsaw, Poland using EasySep ${ }^{\mathrm{TM}}$ Human $\mathrm{CD}^{+}$or $\mathrm{CD}^{+}{ }^{+}$-Cell Isolation Kit (STEMCELL Technologies) according to the manufacturer's protocols. EPCs were isolated from the spleens of anemic mice or human bone marrow aspirates using EasySep ${ }^{\mathrm{TM}}$ Release Mouse Biotin Positive Selection Kit (STEMCELL Technologies) according to the manufacturer's protocols. Biotin-conjugated anti-CD71 antibodies (anti-mouse clone 8D3, NovusBio, antihuman clone DF1513, NovusBio) were used at a final concentration of $1 \mu \mathrm{g} / \mathrm{ml}$. EPCs purity was $>80 \%$. For cell proliferation assay, T-cells were labelled with Cell Trace Violet (CTV) dye (Thermofisher Scientific) at a final concentration of $5 \mu \mathrm{M}$, according 
526 to the manufacturer's manual. Next, the labelled T-cells were plated in L-arginine-free

527 RPMI-medium (SILAC RPMI-medium, Thermofisher Scientific) supplemented with

$528150 \mu \mathrm{M}$ L-arginine and stimulated with Dynabeads T-Activator CD3/CD28 (ratio 1:2,

529 Thermofisher Scientific). The arginase inhibitor OAT-1746 (500 nM), L-arginine (1000

$530 \mu \mathrm{M})$, or $\mathrm{N}$-acetylcysteine $(100 \mu \mathrm{M})$ were added as indicated in the figures. Cells were

531 stained and analyzed by flow cytometry after $72 \mathrm{~h}$ for murine cells and $120 \mathrm{~h}$ for

532 human cells.

\section{Reactive oxygen species (ROS) detection}

The level of ROS in cells was determined using CellROX Green Reagent

(Thermofisher Scientific) or 2',7'-dichlorodihydrofluorescein diacetate (DCFDA). Cells

were stained with CellROX at a final concentration of $5 \mu \mathrm{M}$ or DCFDA at a final

washes with PBS. $\mathrm{H}_{2} \mathrm{O}_{2}$-treated cells served as positive controls. For some experiments, cells stained with CellROX or DCFDA were further stained with

540 fluorochrome-labelled antibodies on ice. Stained cells were acquired on Fortessa X20

541 flow cytometer (BD Biosciences).

542

543

\section{Arginase activity assay and Griess test}

Recombinant enzymes (ARG1 and ARG2) to study ARGi were produced at OncoArendi Therapeutics in E. coli expression system. The proteins were purified by FPLC and stored at $-80^{\circ} \mathrm{C}$ in the storage buffer containing: $20 \mathrm{mM}$ Tris $\mathrm{pH} 8.0$, $100 \mathrm{mM} \mathrm{NaCl}, 10 \mathrm{mM}$ DDT and 10\% glycerol. Basic assay buffer was composed of $100 \mathrm{mM}$ sodium phosphate buffer, $130 \mathrm{mM}$ sodium chloride, $1 \mathrm{mg} / \mathrm{mL}$ BSA, pH 7.4. The enzymatic reaction was carried out in the presence of $200 \mu \mathrm{M} \mathrm{MnCl}_{2}$ (cofactor) and $10 \mathrm{mM}$ or $20 \mathrm{mM}$ L-arginine hydrochloride (for hARG1 or hARG2, respectively), mixed together at the final volume of $25 \mu \mathrm{L}$. Basic developing buffer contained 50 
$551 \mathrm{mM}$ boric acid, $1 \mathrm{M}$ sulfuric acid, 0.03\% (m/v) Brij ${ }^{\circledR} 35$ detergent. $\mathrm{PHZ}$ or $\mathrm{ABH}$ was

552 diluted in basic assay buffer at the volume of $50 \mu \mathrm{L}$. Recombinant enzyme was

553 diluted in basic assay buffer at the volume of $25 \mu \mathrm{L}$. The reaction was performed at

554 the final volume of $100 \mu \mathrm{L}$. Developing mixture included freshly prepared equal

555 volume mixture of developing solution A (4 mM o-phthaldialdehyde) and solution B (4

$556 \mathrm{mM} \mathrm{N}$-(1-naphthyl)ethylenediamine dihydrochloride) prepared in the basic developing

557 buffer. The compound background wells contained each of the tested compound and

558 the substrate/cofactor mixture, but not the recombinant enzyme (data were excluded

559 from the analysis when the compound background exceeded $10 \%$ of the signal

560 obtained in the wells with enzyme). The " $0 \%$ activity" background wells contained

561 only the substrate/cofactor mixture. Following $1 \mathrm{~h}$ incubation at $37^{\circ} \mathrm{C}$, freshly

562 prepared developing reagent was added $(150 \mu \mathrm{L})$ and the colorimetric reaction was

563 developed (12 $\mathrm{min}$ at RT, gentle shaking). The absorbance, proportional to the

564 amount of the produced urea, was measured at $515 \mathrm{~nm}$ using Tecan's Spark ${ }^{\mathrm{TM}}$

565 microplate reader. Data were normalized by referring the absorbance values to the 566 positive control wells $\left(100 \%\right.$ enzyme activity). IC $_{50}$ value was determined by the

567 nonlinear regression method. Arginase activity in the EPCs or splenocytes lysates

568 and cell supernatant was determined using Arginase Activity Assay (Sigma)

569 according to the manufacturer's protocol.

570 To evaluate nitric oxide (NO) production as a measure of NOS (nitric oxide

571 synthase) activity, Griess Reagent System (Promega) was used according to the

572 manufacturer's protocol. Splenocytes or EPCs were isolated from murine spleens

573 and were cultured in non-adherent 6 -well plate $1 \times 10^{6}$ or $5 \times 10^{5}$ cells per $2 \mathrm{ml}$,

574 respectively, for $24 \mathrm{~h}$ followed by supernatants collection.

\section{Bioinformatical analysis of arginase structure}


576 The structure and predicted binding energies for the complexes of $\mathrm{PHZ}$, L-arginine and 2-amino-6-borono-2-(2-(piperidin-1-yl)ethyl)hexanoic acid with both human and mouse arginases were compared. The 3D models of mouse arginases were proposed using available structures of human arginases $(\mathrm{pdb} \mid 4 \mathrm{hww}$ and $\mathrm{pdb} \mid 4 \mathrm{hze}$ for ARG1 and ARG2, respectively) as templates. Both templates shared more than $87 \%$ sequence identity with their respective target. The sequence to structure alignments between mouse arginases and selected templates were calculated with the muscle program $^{47}$. The 3D structure was proposed with MODELLER ${ }^{48}$. Models quality was assessed with the Molprobity webserver ${ }^{49}$. Next, both human and mouse proteins were prepared for docking using the Chimera dock prep module. Molecular docking was carried out with two programs - GOLD 50 and Surflex ${ }^{51}$. The active site was specified based on the position of the inhibitor present in the active site of the arginase 1 (pdb|4hww). The default parameters of both programs were used.

To assess if $\mathrm{PHZ}$ remains stably bound to the active site of both human arginases short molecular dynamics simulations were performed. The initial configurations of ligand-protein complexes were derived from docking results for PHZ. For the PHZ-arginase complexes the simulation included the following steps. First protein and ligand were put in a dodecahedron box with the distance between solute and a box equal to $1 \mathrm{~nm}$. The $0.1 \mathrm{M} \mathrm{NaCl}$ was added to the system including neutralizing counterions. After energy minimization using steepest descent algorithm, 100 ps NVT and NPT simulation were carried out. For this modified Berendsen thermostat was used to maintain the temperature at $310 \mathrm{~K}$ using and Berendsen barostat to keep the pressure at 1 atm. Positions of both protein and ligand heavy 599 atoms remained constrained. During the following 300 ps of simulation time the 600 ligand's constraints were gradually removed. Finally, an unconstrained 100 ns 
601

602

603

604

605

606

607

608

609

610

611

612

613

614

615

616

617

618

619

620

621

622

623

624

625

simulation is performed in which Berendsen barostat was replaced by ParrinelloRahman barostat. During simulation short-range nonbonded interactions were cut off at $1.4 \mathrm{~nm}$, with long-range electrostatics calculated using the particle mesh Ewald (PME) algorithm. Bonds were constrained using the lincs algorithm. Simulations were carried out with Gromacs ${ }^{52}$ using the gromos54a7 force field, modified to include parameters for $\mathrm{Mn}^{2+}$ ion adopted from ${ }^{53}$. Spc model was used for water molecules. Parameters for the ligand were obtained with Automated Topology Builder (ATB $)^{54}$.

Additional analyses were performed to assess if $\mathrm{PHZ}$ can migrate to the arginase active site when present in solute in high concentration. For this analysis protein was put in dodecahedron box with the distance between solute and a box equal to $1.5 \mathrm{~nm}$ in which $6 \mathrm{PHZ}$ molecules were placed randomly. This correspond to $0.02 \mathrm{M}$ concentration of the compound. A similar simulation setup to one described above was used with exception that ligand molecules remained unconstrained throughout simulation.

\section{Protein carbonylation assay}

Carbonyl content of proteins was determined in a 2,4-DNPH reaction. Five $\mu \mathrm{g}$ of murine ARG1 (Cloud-System Corp) was resuspended in $400 \mu$ of distilled water and incubated with $\mathrm{PHZ}(10 \mu \mathrm{M}), \mathrm{PHZ}(10 \mu \mathrm{M})$ with $\mathrm{NAC}(10 \mathrm{mM}), \mathrm{H}_{2} \mathrm{O}_{2}(10 \mathrm{mM})$ or water (negative control) as indicated in the Fig. $3 \mathrm{~d}$ for 1 hour at $37^{\circ} \mathrm{C}$. Proteins were precipitated with $10 \%$ TCA. The precipitates were treated with either $2 \mathrm{~N} \mathrm{HCl}$ alone (control) or $2 \mathrm{~N} \mathrm{HCl}$ containing $5 \mathrm{mg} / \mathrm{ml}$ 2,4-DNPH at RT for $30 \mathrm{~min}$. The resulting hydrazones were precipitated in 10\% TCA and then washed three times with ethanolethyl acetate (1:1). Final precipitates were dissolved in $8 \mathrm{M}$ guanidine chloride. Equal amounts of proteins were separated on $4-12 \%$ SDS-polyacrylamide gel (Bio-Rad), transferred onto nitrocellulose membranes (Bio-Rad) blocked with TBST [Tris- 
buffered saline $(\mathrm{pH} 7.4)$ and $0.05 \%$ Tween 20] supplemented with $5 \%$ non-fat milk. Anti-DNP antibodies (Life Diagnostics, Inc) at concentration $1 \mathrm{U} / \mathrm{ml}$ were used for overnight incubation at $4^{\circ} \mathrm{C}$. After washing with TBST, the membranes were incubated with horseradish peroxidase-coupled secondary antibodies (Jackson Immunores.). The reaction was developed using SuperSignal ${ }^{\mathrm{TM}}$ West Femto Maximum Sensitivity Substrate (ThermoFisher Scientific) and imaged using ChemiDoc Touch Gel Imaging System (Bio-Rad). Densitometry was done using ImageJ software.

RNA isolation from EPCs, reverse transcription, and quantitative polymerase chain reaction

Total RNA was isolated from EPCs isolated from murine spleens using RNeasy Mini Kit (Qiagen). RNA was subjected to reverse transcription using GoScript ${ }^{\mathrm{TM}}$ Reverse Transcriptase system (Promega). All qPCRs were performed in MicroAmp Fast Optical 96 WellReaction Plates (Thermo Fisher Scientific) using AppliedBiosystems 7500 Fast Real-Time PCR System with 7500Software V2.0.6 (Thermo Fisher Scientific). Samples were assayed in triplicates. Primers sequences used in the study: ARG1 forward 5'- CTCCAAGCCAAAGTCCTTAGAG-3', reverse 5'AGGAGCTGTCATTAGGGACATC-3', ARG2 forward 5'AGGAGTGGAATATGGTCCAGC-3', reverse 5'-GGGATCATCTTGTGGGACATT-3', and GAPDH forward 5'-GAAGGTGGTGAAGCAGGCATC-3', reverse 5'GCATCGAAGGTGGAAGAGTGG-3' as an endogenous control. The mean Ct values of a target gene and endogenous control were used to calculate relative expression using the $2^{-\Delta C t}$ method.

\section{Western blot}


650 Splenocytes lysates were prepared using Cell Lysis Buffer (\#9803, CellSignaling

651

652

653

654

655

656

657

658

659

660

661

662

663

664

665

666

667

668

669

670

671

672

673

674

Technology) supplemented with protease inhibitors (Roche) according to the manufacturer's protocol. Equal amounts of proteins samples were boiled in Laemmli loading buffer, separated on 4-12\% SDS-polyacrylamide gel (Biorad), transferred onto nitrocellulose membranes (Bio-Rad) blocked with TBST [Tris-buffered saline (pH 7.4) and $0.05 \%$ Tween 20] supplemented with 5\% non-fat milk. Anti-Arg1 antibodies (polyclonal, GTX109242, GeneTex) at dilution 1:2000 or anti-Arg2 antibodies (polyclonal, ab81505, Abcam) at dilution 1:1000 were used for overnight incubation at $4^{\circ} \mathrm{C}$. After washing with TBST, the membranes were incubated with horseradish peroxidase-coupled secondary antibodies (Jackson Immunores.). The reaction was developed using SuperSignal ${ }^{\mathrm{TM}}$ West Femto Maximum Sensitivity Substrate (ThermoFisher Scientific) and imaged using ChemiDoc Touch Gel Imaging System (Bio-Rad). After imaging, bound antibodies were removed from membranes using Restore $^{\mathrm{TM}}$ PLUS Western Blot Stripping Buffer (ThermoFisher Scientific), followed by blocking with TBST supplemented with $5 \%$ non-fat milk Next, the membranes were incubated with anti- $\beta-A c t i n$ (A5060, SantaCruz) conjugated with peroxidase. Densitometry was done using ImageJ software.

\section{Erythroid cells differentiation}

EPCs were differentiated from human peripheral blood mononuclear cells (PBMC) according to the protocol by Heshusius et al. ${ }^{55}$ with modifications. Human PBMC were purified from buffy coats from healthy donors by density separation using Lymphoprep (STEMCELL Technologies). PBMC were seeded at $10 \times 10^{6}$ cells $/ \mathrm{mL}$ in erythroid differentiation-promoting medium based on StemSpan ${ }^{\mathrm{TM}}$ Serum-Free Expansion Medium (SFEM) supplemented with human recombinant EPO (2 U/ml, Roche), human recombinant stem cell factor (25 ng/ml, R\&DSystems), 
675 dexamethasone (1 $\mu \mathrm{M}$, SigmaAldrich), human recombinant insulin $(10 \mathrm{ng} / \mathrm{ml}$, 676 SigmaAldrich), L-Glutamine (2 mM, SigmaAldrich), sodium pyruvate (1 mM, Gibco), 677 MEM non-essential amino acids (1x, Gibco), bovine serum albumin $(0.1 \% \mathrm{~m} / \mathrm{v})$, 678 SigmaAldrich), EmbryoMax Nucleosides (1x, Merck), and $100 \mathrm{U} / \mathrm{ml}$ penicillin and 100 $679 \mu \mathrm{g} / \mathrm{ml}$ streptomycin (Sigma-Aldrich). The expansion and differentiation of EPCs were 680 determined by flow cytometry.

681

682

683

684

685

686

687

688

689

690

691

692

693

694

695

696

697

\section{Statistical analysis}

Data are shown as means \pm SD or means \pm SEM, as indicated in the figure legends. Graphpad Prism 8.4.3 (GraphPad Software) was used for statistical analyses. Data distribution was tested using Shapiro-Wilk test. Statistical analyses of three or more groups were compared using one-way analysis of variance (ANOVA) followed by Tukey's, Dunnett's or Bonferroni's multiple comparisons test or Kruskal-Wallis test followed by Dunn's multiple comparisons test. Statistical analyses of two groups were compared using unpaired $t$-test or Mann-Whitney test.

\section{Acknowledgements}

This work has been co-supported by grants iONKO (Regionalna Inicjatywa Doskonalosci) from the Polish Ministry of Science and Higher Education (J.G.), 2019/35/B/NZ6/00540 (D.N.), 2017/25/B/NZ6/01139 (J.G.), and 2016/23/B/NZ6/03463 (D.N.) from the National Science Center in Poland. D.P. is financed by TEAM program from the Foundation for Polish Science co-financed by the European Union under the European Regional Development Fund as well as grants 2019/35/O/ST6/02484 and 2020/37/B/NZ2/03757 from the National Science Center in Poland. M.L. is funded by IDUB against COVID-19 project granted by the 
Warsaw University of Technology under the program Excellence Initiative: Research University (IDUB). Some elements of the figures were generated with Biorender.com.

\section{Authorship Contributions}

TM.G. designed and supervised the study, conducted the experiments, analyzed the data, and wrote the manuscript. A.S. participated in in vivo studies,. Z.R. participated in in vitro experiments. M.L. and D.P. performed molecular docking and molecular dynamics simulations, K.K. performed real-time qPCR and participated in in vitro experiments, M.M. and O.C. collected and provided human blood samples, A.R.-L. performed analysis of murine blood, M.J. participated in in vitro experiments, P.P and M.M.G. carried out arginase activity assays, R.B. designed and synthesized OAT1746, M.W. bred and provided Arg2 $\%$ mice, A.T and G.B. collected and provided HSPCs. J.G. conceived, designed and supervised the study, provided funding and wrote the manuscript. D.N. provided funding, performed in vivo studies, designed and supervised the study, and wrote the manuscript. All authors edited and approved the final manuscript.

\section{Disclosure of Conflicts of Interest}

P.P., M.M.G., and R.B. are employees of OncoArendi Therapeutics, Warsaw, Poland.

\section{References}

1. Hom J, Dulmovits BM, Mohandas N, Blanc L. The erythroblastic island as an emerging paradigm in the anemia of inflammation. Immunologic Research 63, 75-89 (2015).

2. Elahi S. Neglected Cells: Immunomodulatory Roles of CD71+ Erythroid Cells. Trends in Immunology 40, 181-185 (2019). 
3. Shokrollah E, Siavash M. Immunological consequences of extramedullary erythropoiesis: immunoregulatory functions of CD71+ erythroid cells. Haematologica 105, 1478-1483 (2020).

4. Elahi S, et al. Immunosuppressive CD71+ erythroid cells compromise neonatal host defence against infection. Nature 504, 158 (2013).

5. Namdar A, Koleva P, Shahbaz S, Strom S, Gerdts V, Elahi S. CD71+ erythroid suppressor cells impair adaptive immunity against Bordetella pertussis. Scientific Reports 7, 7728 (2017).

6. Yang L, et al. Regulation of bile duct epithelial injury by hepatic CD71+ erythroid cells. JCl Insight 5, (2020).

7. Delyea C, et al. CD71(+) Erythroid Suppressor Cells Promote Fetomaternal Tolerance through Arginase-2 and PDL-1. J Immunol 200, 4044-4058 (2018).

8. Chen J, et al. Intratumoral CD45+CD71+ erythroid cells induce immune tolerance and predict tumor recurrence in hepatocellular carcinoma. Cancer Letters, (2020).

9. Zhao L, et al. Late-stage tumors induce anemia and immunosuppressive extramedullary erythroid progenitor cells. Nature Medicine 24, 1536-1544 (2018).

10. Shim YA, Weliwitigoda A, Campbell T, Dosanjh M, Johnson P. Splenic erythroid progenitors decrease TNFa production by macrophages and reduce systemic inflammation in a mouse model of T cell-induced colitis. European Journal of Immunology n/a, (2020).

11. Namdar A, et al. CD71<sup >+</sup $>$ Erythroid Cells Exacerbate HIV-1 Susceptibility, Mediate <em>trans</em>-Infection, and Harbor Infective Viral Particles. mBio 10, e02767-02719 (2019).

12. Shahbaz S, et al. Erythroid precursors and progenitors suppress adaptive immunity and get invaded by SARS-CoV-2. bioRxiv, 2020.2008.2018.255927 (2020).

13. Chen K, Liu J, Heck S, Chasis JA, An X, Mohandas N. Resolving the distinct stages in erythroid differentiation based on dynamic changes in membrane protein expression during erythropoiesis. Proc Natl Acad Sci U S A 106, 17413-17418 (2009). 
14. Elahi S, et al. CD71+ Erythroid Cells in Human Neonates Exhibit Immunosuppressive Properties and Compromise Immune Response Against Systemic Infection in Neonatal Mice. Frontiers in Immunology 11, (2020).

15. Rodriguez PC, et al. Arginase I production in the tumor microenvironment by mature myeloid cells inhibits T-cell receptor expression and antigen-specific Tcell responses. Cancer research 64, 5839-5849 (2004).

16. Modolell $\mathrm{M}$, et al. Local suppression of $\mathrm{T}$ cell responses by arginase-induced L-arginine depletion in nonhealing leishmaniasis. PLoS Negl Trop Dis 3, e480e480 (2009).

17. Van Zandt MC, et al. Discovery of (R)-2-amino-6-borono-2-(2-(piperidin-1$\mathrm{yl}$ )ethyl)hexanoic acid and congeners as highly potent inhibitors of human arginases I and II for treatment of myocardial reperfusion injury. Journal of medicinal chemistry 56, 2568-2580 (2013).

18. Itano HA, Hirota K, Hosokawa K. Mechanism of induction of haemolytic anaemia by phenylhydrazine. Nature 256, 665-667 (1975).

19. Czystowska-Kuzmicz M, et al. Small extracellular vesicles containing arginase-1 suppress T-cell responses and promote tumor growth in ovarian carcinoma. Nature communications 10, 3000 (2019).

20. Shi O, Morris SM, Jr., Zoghbi H, Porter CW, O'Brien WE. Generation of a mouse model for arginase II deficiency by targeted disruption of the arginase II gene. Mol Cell Biol 21, 811-813 (2001).

21. Rodriguez PC, Zea AH, Culotta KS, Zabaleta J, Ochoa JB, Ochoa AC. Regulation of T cell receptor CD3zeta chain expression by L-arginine. J Biol Chem 277, 21123-21129 (2002).

22. Chénais B, Molle I, Trentesaux C, Jeannesson P. Time-course of butyric acidinduced differentiation in human K562 leukemic cell line: rapid increase in Yglobin, porphobilinogen deaminase and NF-E2 mRNA levels. Leukemia 11, 1575-1579 (1997).

23. Moras M, Lefevre SD, Ostuni MA. From Erythroblasts to Mature Red Blood Cells: Organelle Clearance in Mammals. Frontiers in Physiology 8, (2017).

24. $\mathrm{Hu} \mathrm{J}$, et al. Isolation and functional characterization of human erythroblasts at distinct stages: implications for understanding of normal and disordered erythropoiesis in vivo. Blood 121, 3246-3253 (2013). 
25. Nandakumar SK, Ulirsch JC, Sankaran VG. Advances in understanding erythropoiesis: evolving perspectives. Br J Haematol 173, 206-218 (2016).

26. Grzywa TM, et al. Myeloid Cell-Derived Arginase in Cancer Immune Response. Front Immunol 11, 938 (2020).

27. Zea $\mathrm{AH}$, et al. Arginase-producing myeloid suppressor cells in renal cell carcinoma patients: a mechanism of tumor evasion. Cancer Res 65, 30443048 (2005).

28. Köstlin N, et al. Granulocytic myeloid derived suppressor cells expand in human pregnancy and modulate T-cell responses. European Journal of Immunology 44, 2582-2591 (2014).

29. Otsuji M, Kimura Y, Aoe T, Okamoto Y, Saito T. Oxidative stress by tumorderived macrophages suppresses the expression of CD3 zeta chain of T-cell receptor complex and antigen-specific T-cell responses. Proc Natl Acad Sci U $S$ A 93, 13119-13124 (1996).

30. Reade MC, Weissfeld L, Angus DC, Kellum JA, Milbrandt EB. The prevalence of anemia and its association with 90-day mortality in hospitalized communityacquired pneumonia. BMC Pulm Med 10, 15 (2010).

31. Liu L, et al. Multiple myeloma hinders erythropoiesis and causes anaemia owing to high levels of CCL3 in the bone marrow microenvironment. Scientific Reports 10, 20508 (2020).

32. Musallam KM, et al. Preoperative anaemia and postoperative outcomes in non-cardiac surgery: a retrospective cohort study. The Lancet 378, 1396-1407 (2011).

33. Dunne JR, Malone D, Tracy JK, Gannon C, Napolitano LM. Perioperative anemia: an independent risk factor for infection, mortality, and resource utilization in surgery. J Surg Res 102, 237-244 (2002).

34. Hill DL, et al. Immune system development varies according to age, location, and anemia in African children. Sci Transl Med 12, (2020).

35. Peter V, et al. Normal and pathological erythropoiesis in adults: from gene regulation to targeted treatment concepts. Haematologica 103, 1593-1603 (2018).

36. Han $\mathrm{Y}$, et al. Tumor-Induced Generation of Splenic Erythroblast-like Ter-Cells Promotes Tumor Progression. Cell 173, 634-648.e612 (2018). 
37. Dunsmore G, Bozorgmehr N, Delyea C, Koleva P, Namdar A, Elahi S. Erythroid Suppressor Cells Compromise Neonatal Immune Response against $<$ em>Bordetella pertussis $<$ /em>. The Journal of Immunology, ji1700742 (2017).

38. Paulson RF, Ruan B, Hao S, Chen Y. Stress Erythropoiesis is a Key Inflammatory Response. Cells 9, (2020).

39. Greten FR, Grivennikov SI. Inflammation and Cancer: Triggers, Mechanisms, and Consequences. Immunity 51, 27-41 (2019).

40. Dunsmore $\mathrm{G}$, et al. Lower Abundance and Impaired Function of CD71+ Erythroid Cells in Inflammatory Bowel Disease Patients During Pregnancy. $J$ Crohns Colitis 13, 230-244 (2019).

41. Libregts SF, et al. Chronic IFN-y production in mice induces anemia by reducing erythrocyte life span and inhibiting erythropoiesis through an IRF1/PU.1 axis. Blood 118, 2578-2588 (2011).

42. de Bruin AM, Voermans C, Nolte MA. Impact of interferon- $y$ on hematopoiesis. Blood 124, 2479-2486 (2014).

43. Srikantia SG, Prasad JS, Bhaskaram C, Krishnamachari KA. Anaemia and immune response. Lancet (London, England) 1, 1307-1309 (1976).

44. Aly SS, Fayed HM, Ismail AM, Abdel Hakeem GL. Assessment of peripheral blood lymphocyte subsets in children with iron deficiency anemia. $B M C$ Pediatrics 18, 49 (2018).

45. Pasricha S-R, Colman K, Centeno-Tablante E, Garcia-Casal M-N, PeñaRosas J-P. Revisiting WHO haemoglobin thresholds to define anaemia in clinical medicine and public health. The Lancet Haematology 5, e60-e62 (2018).

46. O'Connell KE, et al. Practical murine hematopathology: a comparative review and implications for research. Comp Med 65, 96-113 (2015).

47. Edgar RC. MUSCLE: multiple sequence alignment with high accuracy and high throughput. Nucleic Acids Res 32, 1792-1797 (2004).

48. Webb B, Sali A. Comparative Protein Structure Modeling Using MODELLER. Curr Protoc Bioinformatics 54, 56 1-5 637 (2016). 
49. Williams CJ, et al. MolProbity: More and better reference data for improved allatom structure validation. Protein Sci 27, 293-315 (2018).

50. Jones G, Willett $P$, Glen RC, Leach AR, Taylor R. Development and validation of a genetic algorithm for flexible docking. J Mol Biol 267, 727-748 (1997).

51. Jain AN. Surflex: fully automatic flexible molecular docking using a molecular similarity-based search engine. Journal of medicinal chemistry 46, 499-511 (2003).

52. Pronk S, et al. GROMACS 4.5: a high-throughput and highly parallel open source molecular simulation toolkit. Bioinformatics 29, 845-854 (2013).

53. M. Bradbrook G, et al. X-Ray and molecular dynamics studies of concanavalin-A glucoside and mannoside complexes Relating structure to thermodynamics of binding. Journal of the Chemical Society, Faraday Transactions 94, 1603-1611 (1998).

54. Malde AK, et al. An Automated Force Field Topology Builder (ATB) and Repository: Version 1.0. J Chem Theory Comput 7, 4026-4037 (2011).

55. Heshusius S, et al. Large-scale in vitro production of red blood cells from human peripheral blood mononuclear cells. Blood Advances 3, 3337-3350 (2019). 
a, Representative plots of CD71+TER119+ EPCs in the spleens of control, anemic and 3-days old neonatal mice. b, The frequency of CD71+TER119+ EPCs in the spleens of control $(n=10)$, control-IgG $(n=7)$, anemic (NHA, $n=13 ; H A-P H Z, n=9 ; H A-$ TER119, $n=8)$, and 3-days old neonatal mice $(n=5)$. $P$ values calculated with KruskalWallis test with Dunn's post-hoc test. c, Number of CD71+TER119+ EPCs in the spleens of control $(n=4)$, anemic (NHA, $n=4 ; H A-P H Z, n=4 ; H A-T E R 119, n=4)$, and neonatal mice. $P$ values calculated with one-way ANOVA with Dunnet's post-hoc test. d, Percentages of CD45.2 $2^{-}$and CD45.2+ cells within EPCs (CD71+TER119+) expression and cells size ${ }^{13} . \mathbf{f}$, Developmental stages of EPCs in control mice $(n=9)$, NHA mice $(n=13)$, HA-PHZ $(n=9)$, HA-TER119 $(n=5)$, and neonatal mice $(n=5)$. Data show means \pm SD. Each point in $\mathbf{b}$-d represents data from individual mice. $n$ values are the numbers of mice used to obtain the data. The source data underlying Fig.1b$d, f$ are provided as a Source Data file.

\section{Figure 2. Anemic mice have impaired T-cell immune response}

a, Schematic presentation of the experimental setting. T-cells isolated from OT-I mice were labelled with CellTraceViolet (CTV) and adoptively transferred to anemic and OT-I T-cells in the spleen of NHA mice $(n=8)$, HA-PHZ mice $(n=8)$, and healthy controls $(\mathrm{n}=5)$. Histograms show the fluorescence of CTV (CellTraceViolet) - V450. $P$ values calculated with one-way ANOVA with Tukey's post-hoc test. c, Representative 
949

950

951

952

953

954

955

956

957

958

959

960

961

962

963

964

965

966

967

968

969

970

971

972

973

$\mathrm{CD}^{+}{ }^{+}$-cells co-cultured with EPCs isolated from the spleens of NHA $(n=8), H A-P H Z$ $(\mathrm{n}=8)$, HA-TER119 $(\mathrm{n}=4)$. T-cell:EPC ratio was 1:2. Representative proliferation histograms of aCD3/aCD28-stimulated CD4 ${ }^{+}$T-cells co-cultured with EPCs. Histograms show the fluorescence of CTV (CellTraceViolet) - V450. $P$ values calculated with one-way ANOVA with Dunnet's post-hoc test. Data show means \pm SD. Each point in $\mathbf{b}, \mathbf{d}$ represents data from individual mice. $n$ values are the numbers of mice. The source data underlying Fig. 2b,d are provided as a Source Data file.

\section{Figure 3. EPCs express ARG2 and have high levels of ROS}

a, Mean Fluorescence Intensity (MFI) of CellROX Green - FITC in EPCs $\left(\mathrm{CD} 71^{+} \mathrm{TER}_{\left.119^{+}\right)}\right.$and RBCs $\left(\mathrm{CD} 71^{-T E R} 119^{+}\right)$of control mice $(\mathrm{n}=6)$ and NHA $(\mathrm{n}=6)$ and HA-PHZ (n=6). Histograms show the representative fluorescence of CellROX Green - FITC in EPCs from the spleen of NHA mouse. $P$ values calculated with unpaired t-test. b, Mean Fluorescence Intensity (MFI) of CellROX Green - FITC in EPCs, leukocytes $\left(C D 45^{+}\right)$, T-cells $\left(C D 45^{+}\right.$CD3e $\left.e^{+}\right)$, myeloid cells $\left(C D 45^{+} C D 11 b^{+}\right)$ $(\mathrm{n}=18)$. $P$ values calculated with one-way ANOVA with Dunnet's post-hoc test. c, Percentages of ARG2+ EPCs in control mice $(n=11)$, anemic mice (NHA, $n=5 ; H A-$ PHZ, $n=11$; HA-TER119, $n=11)$, neonatal mice $(n=5)$, and isotype control-IgG-treated mice (control-lgG, $n=7$ ). d, Percentages of ARG1+ EPCs based on intracellular staining $(\mathrm{n}=5)$. $P$ values calculated with one-way ANOVA with Dunnet's post-hoc test and with unpaired t-test for HA-TER119. e, Percentages of YFP+ EPCs in reporter

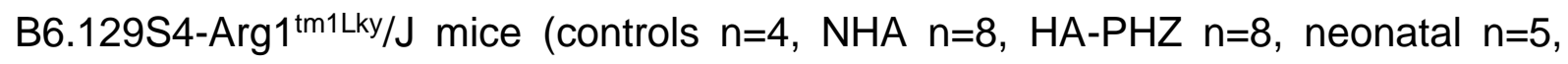
control-lgG $n=4$, HA-TER119 $n=8)$. $P$ values calculated with one-way ANOVA with Dunnet's post-hoc test and with unpaired t-test for HA-TER119. f, Mean Fluorescence Intensity (MFI) of YFP - FITC in EPCs of reporter B6.129S4-

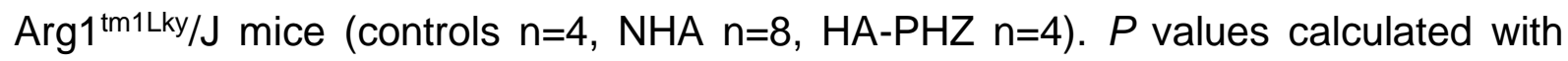


one-way ANOVA with Dunnet's post-hoc test. $\mathbf{g , h}$, Total arginase activity in EPCs lysates $(\mathbf{g}, \mathrm{n}=8)$ or in the supernatants from EPCs cultures $(\mathbf{h}, \mathrm{n}=8) . P$ values calculated with one-way ANOVA with unpaired t-test. i, Percentages of ARG1+ EPCs isolated from the spleens of B6.129S4-Arg1 ${ }^{\text {tm1Lky } / J}$ incubated with diluent or $\mathrm{PHZ}$ $(100 \mu \mathrm{M}$ for $24 \mathrm{~h})(\mathrm{n}=3)$. $P$ values calculated with one-way ANOVA with unpaired ttest. Data show means \pm SD. Each point in a-i represents data from individual mice. $n$ values are the numbers of mice used to obtain the data. The source data underlying Fig.3a-i are provided as a Source Data file.

\section{Figure 4. Phenylhydrazine targets arginase, inhibits its activity and induces} oxidative damage

a, Inhibition curves for recombinant human $A R G 1$ and $A R G 2$, and $I_{50}$ values for $\mathrm{PHZ}(\mathrm{n}=2)$ and 2(S)-amino-6-boronohexanoic acid $(\mathrm{ABH})$. b, NO production from EPCs and whole splenocytes population isolated from NHA $(n=4)$ and $\mathrm{HA}-\mathrm{PHZ}(\mathrm{n}=4)$ mice. $P$ value was calculated with unpaired $t$-test. c, The electrostatic surface potential of the human ARG1. The potential was calculated with APBS and projected onto the molecular surface of the protein. The figure was prepared with UCSF Chimera. d, Carbonylation of ARG1 in the presence of $\mathrm{PHZ}$ and/or N-acetylcysteine (NAC) (n=3). Representative blot (left) and densitometric analysis done with ImageJ software (right). $P$ value was calculated with Ordinary one-way ANOVA with Dunnett's multiple comparisons test. Data show means \pm SD. Each point in $\mathbf{b}$ represents data from individual mice. $n$ values are the numbers of mice used to obtain the data or number of biological replicates of in vitro experiments. The source data underlying Fig.4, 4b, 4d are provided as a Source Data file.

Figure 5. EPCs degrade L-Arg and produce ROS leading to the suppression of T-cells 
a, Proliferation and surface markers in aCD3/aCD28-stimulated CD4 ${ }^{+}$T-cells cocultured with EPCs isolated from NHA mice $(n=4)$ at a ratio 1:2 (T-cells:EPCs). $P$ value was calculated with unpaired t test. b, Effects of ARGi (OAT-1746, $500 \mathrm{nM})$ and ROSi (N-acetylcysteine, $100 \mu \mathrm{M}$ ) on the proliferation of $\alpha \mathrm{CD} 3 / \alpha \mathrm{CD} 28$-stimulated CD4+ T-cells co-cultured with EPCs isolated from the spleens of NHA mice $(n=4)$. Representative proliferation histograms of aCD3/aCD28-stimulated CD4+ ${ }^{+}$-cells cocultured with EPCs in the presence of ARGi or ROSi. Histograms show the fluorescence of CTV (CellTraceViolet) - V450. $P$ value was calculated with one-way ANOVA with Bonferroni's post-hoc test. c, Effects of L-arginine supplementation $(1000 \mu \mathrm{M})$ or ARGi $(\mathrm{OAT}-1746,500 \mathrm{nM})$ on the proliferation of aCD3/aCD28stimulated $\mathrm{CD}^{+}{ }^{+}$-cells cultured in full medium or in EPCs-conditioned medium (CM) $(\mathrm{n}=3) . P$ value was calculated with one-way ANOVA with Bonferroni's post-hoc test.

d, Proliferation of aCD3/aCD28-stimulated $\mathrm{CD}^{+}{ }^{+}$T-cells co-cultured with EPCs isolated from $\mathrm{NHA} \mathrm{Arg}^{-/-}$mice or NHA wild-type $\operatorname{Arg}^{+/+}$mice at a ratio $1: 4$ (T:cells:EPCs). Representative proliferation histograms of $\alpha C D 3 / \alpha C D 28$-stimulated $\mathrm{CD}^{+}$T-cells co-cultured with EPCs isolated from Arg2 $^{-/-}$mice or wild-type Arg2 $^{+/+}$ mice in the presence of ARGi or ROSi. Histograms shows the fluorescence of CTV (CellTraceViolet) - V450. $P$ value was calculated with one-way ANOVA with Bonferroni's post-hoc test. e, Arginase activity of the splenocytes lysate of control and anemic mice calculated per $\mu \mathrm{g}$ of total protein based on bicinchoninic acid (BCA) 1019 protein assay. $P$ value was calculated with unpaired t-test. $\mathbf{f}$, The level of ARG1 and ARG2 in the splenocytes lysate of control $(n=4)$ and anemic mice $(n=4)$. $\beta$-actin as used as a loading control. g,h Relative density of ARG1 (g) and ARG2 (h) compared to $\beta$-actin. $P$ value was calculated with unpaired t-test. $\mathbf{i}, \mathbf{j}$, The level of $C D 3 \zeta$ in $C D 4^{+}$ (i) and $\mathrm{CD}^{+}(\mathbf{j})$ T-cells in the spleen of control $(n=4)$ and anemic mice $(n=4)$ based 
on intracellular staining. $P$ value was calculated with unpaired t-test. $\mathbf{k}, \mathbf{l}$, The levels of CD3 3 in $\mathrm{CD}^{+}(\mathbf{k})$ and $\mathrm{CD}^{+}(\mathrm{I})$ aCD3/aCD28-stimulated T-cells in the presence of EPCs isolated from anemic mice $(n=4)$ based on intracellular staining. $P$ value was calculated with one-way ANOVA with Bonferroni's post-hoc test. Data show means \pm SD. Each point in a-e, $\mathbf{g}-\mathbf{I}$ represents data from individual mice. $n$ values are the numbers of mice used to obtain the data or number of biological replicates in in vitro experiments. The source data underlying Fig. 5a-I are provided as a Source Data file.

Figure 6. EPCs expand in the blood of anemic patients and suppress T-cells response

a, Percentage of $\mathrm{CD} 71^{+} \mathrm{CD} 235 \mathrm{a}^{+}$EPCs of live cells in the whole blood of non-anemic (controls, $\mathrm{n}=41)$ and anemic patients $(\mathrm{n}=41)$. $P$ value was calculated with Mann Whitney test. b, Representative dot plots of EPCs in the blood of non-anemic and anemic patients. c, EPCs count per $\mu$ l of blood in controls $(n=41)$ and anemic patients $(\mathrm{n}=41)$. $P$ value was calculated with Mann Whitney test. $\mathbf{d}$, Correlation of the number of EPCs per $\mu \mathrm{l}$ of blood and hemoglobin concentration $(n=82)$. Correlation was calculated with Spearman r. e, EPCs count per $\mu \mathrm{l}$ of blood in non-anemic controls $(n=34)$ and patients with mild $(n=14)$, moderate $(n=32)$, and severe $(n=2)$ anemia. $P$ value was calculated with Kruskal-Wallis test with Dunn's post-hoc test. $\mathbf{f}, \mathbf{g}$, Percentage of EPCs in the fraction of peripheral blood mononuclear cells (PBMC) in controls $(n=12)$ and anemic patients $(n=13)(f)$ and representative dot plots of EPCs (g). P value was calculated with Mann-Whitney test. g, PBMC of controls $(n=12)$ and anemic patients $(n=13)$ were stimulated with aCD3/aCD28 for $12 \mathrm{~h}$ in the presence of protein transport inhibitor. IFN-y level was determined by intracellular staining. $P$ value was calculated with unpaired t-test. Data show means \pm SD. Each point in a,c-f,h represents data from individual patients. $n$ values are the 
numbers of patients used to obtain the data or number of biological replicates in in vitro experiments. The source data underlying Fig.6a, 6c-f, $6 \mathrm{~g}$ are provided as a Source Data file.

Figure 7. EPCs from human bone marrow express ARG1 and ARG2 and suppress T-cells proliferation

a, Representative dot plots of $C D 71{ }^{+} \mathrm{CD} 235 \mathrm{a}^{+}$EPCs in the aspirate of human bone marrow. b,c, Representative histograms of ARG2 (b) and ARG1 (c) expression in EPCs from human bone marrow. Fluorescence-minus-one (FMO) showed as unstained controls. $\mathbf{d}, \mathbf{e}$, Proliferation triggered by aCD3/aCD28 of CTV-labelled CD4+ (d) and $\mathrm{CD}^{+}$(e) T-cells co-cultured with EPCs isolated from the human bone marrow. T-cell:EPC ratio was 1:2 $(n=9)$. Proliferation of T-cells in coculture with EPCs was calculated in relation to no-EPCs T-cells proliferation as $100 \%$. $P$ value was calculated with Friedman test with Dunn's post-hoc test. Data show means \pm SD. $n$ values are the numbers of individual patients used to obtain the data or number of biological replicates in in vitro experiments. The source data underlying Fig.7d, 7e are provided as a Source Data file.

\section{Figure 8. Suppression of T-cells is a general feature of erythroid cells that}

\section{diminishes with EPCs maturation}

$\mathbf{a , b}$, Proliferation triggered by aCD3/aCD28 of CTV-labelled CD4+ $(\mathbf{a})$ and $\mathrm{CD}^{+}(\mathbf{b})$ T-cells co-cultured with erythroid cell lines (K562, HEL92.1.7, TF-I). T-cell:erythroid cell ratio was 1:3. Data from one representative experiment out of three. $P$ value was calculated with one-way ANOVA with Bonferroni's post-hoc test. c, Representative density plot of EPCs differentiated from PBMC. d,e, Proliferation triggered by aCD3/aCD28 of CTV-labelled $\mathrm{CD}^{+}(\mathbf{d})$ and $\mathrm{CD}^{+}(\mathbf{e})$ T-cells co-cultured with EPCs 
1073 differentiated from PBMC $(\mathrm{n}=4)$. $P$ value was calculated with one-way ANOVA with

1074 Dunnett's post-hoc test. f, Representative density plots of EPCs differentiation from 1075 PBMC based on CD71 and CD235a expression. g, Proliferation triggered by 1076 aCD3/aCD28 of CTV-labelled CD4 ${ }^{+}$co-cultured with EPCs differentiated from PBMC 1077 at different developmental stages. h,i, Relative proliferation of $\mathrm{CD}^{+}(\mathbf{h})$ and $\mathrm{CD}^{+}(\mathbf{i})$ 1078 T-cells cocultured with EPCs differentiated from PBMC at different timepoints. $P$ 1079 value was calculated with one-way ANOVA with Bonferroni's post-hoc test. j,k, 1080 Levels of CD44 (j), and CD49d (k) during erythroid differentiation from PBMC. Data 1081 show means \pm SD. Each point in $\mathbf{d}$, $\mathbf{h}-\mathbf{k}$ represents data from individual patients. $n$ 1082 values are the numbers of individual patients used to obtain the data or number of 1083 biological replicates in in vitro experiments. The source data underlying Fig.8a, 8b, 1084 8d-, 8h-k are provided as a Source Data file. 
bioRxiv preprint doi: https://doi.org/10.1101/2021.01.18.427109; this version posted January 19, 2021. The copyright holder for this preprint (which was not certified by peer review) is the author/funder, who has granted bioRxiv a license to display the preprint in perpetuity. It is made available under aCC-BY-NC-ND 4.0 International license.

Figure 1.

a

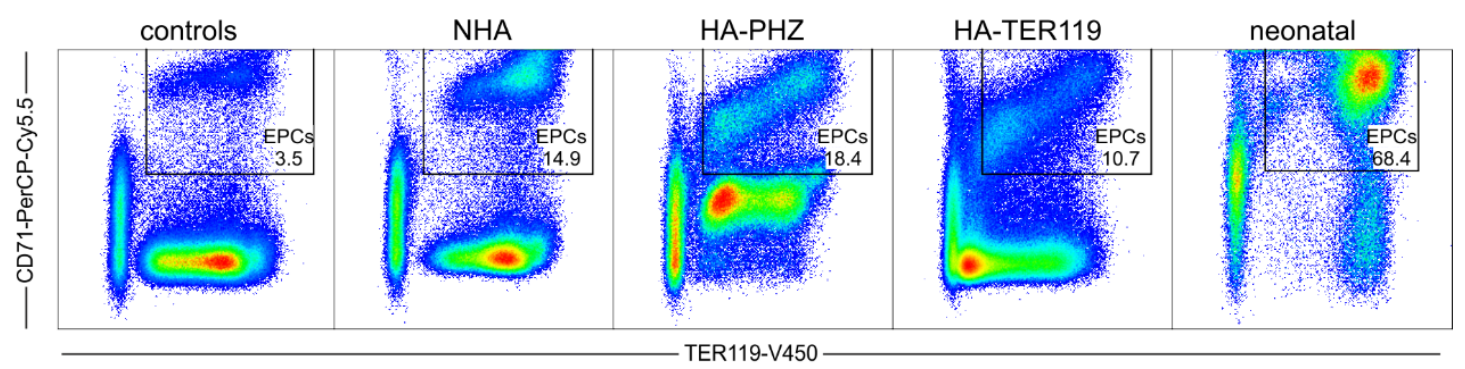

b

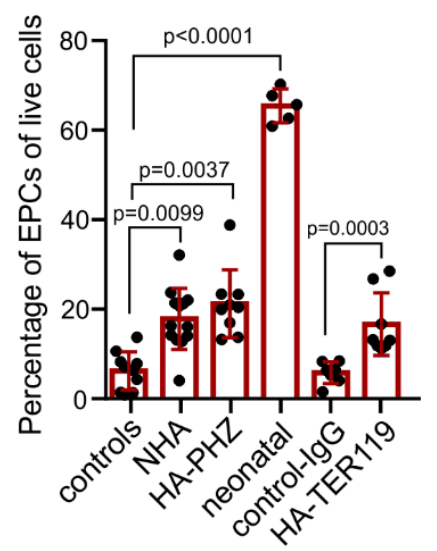

e

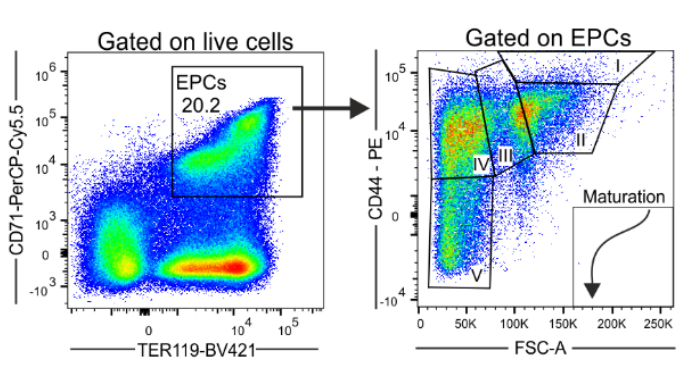

1087
C

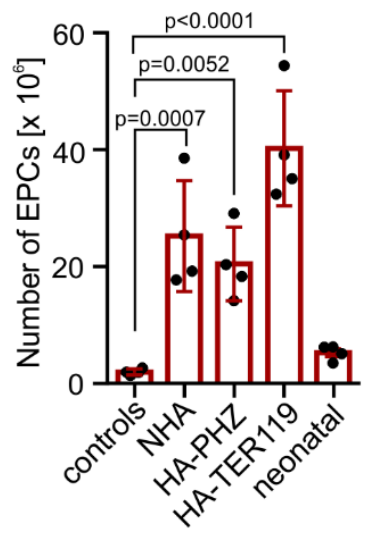

d

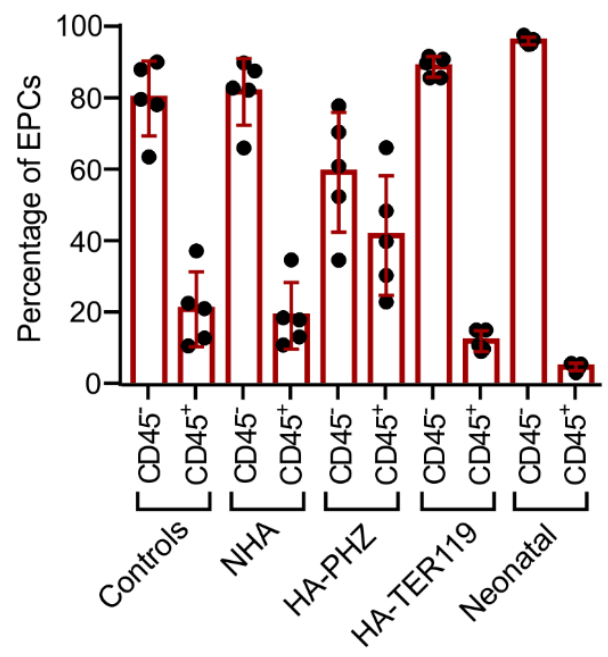

\section{f}
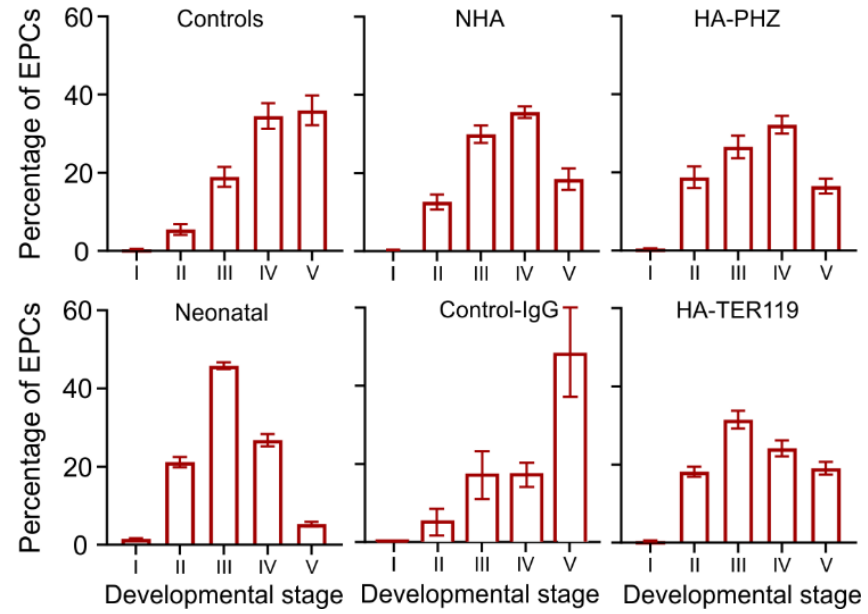
bioRxiv preprint doi: https://doi.org/10.1101/2021.01.18.427109; this version posted January 19, 2021. The copyright holder for this preprint (which was not certified by peer review) is the author/funder, who has granted bioRxiv a license to display the preprint in perpetuity. It is made available under aCC-BY-NC-ND 4.0 International license.

\section{Figure 2.}

a
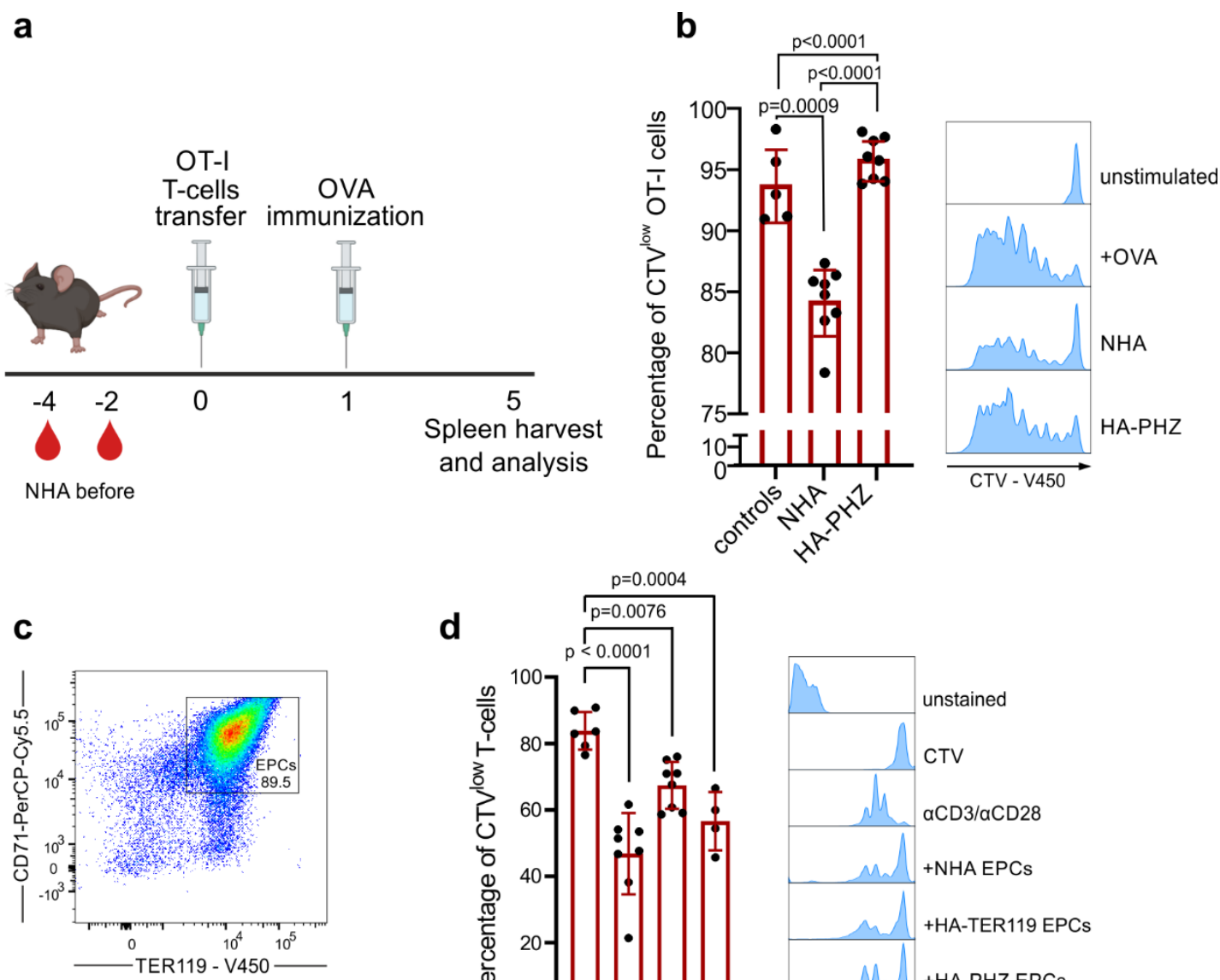

1090

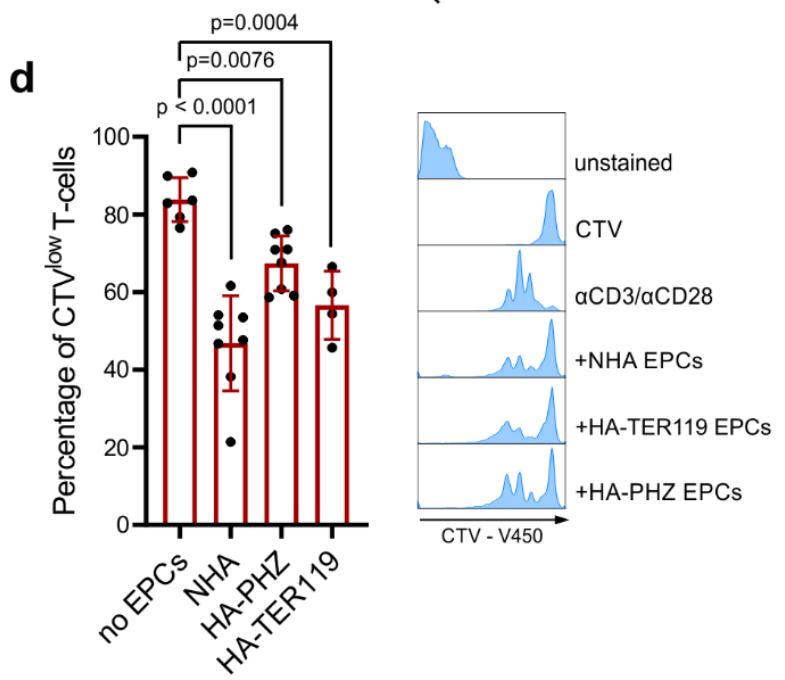


a

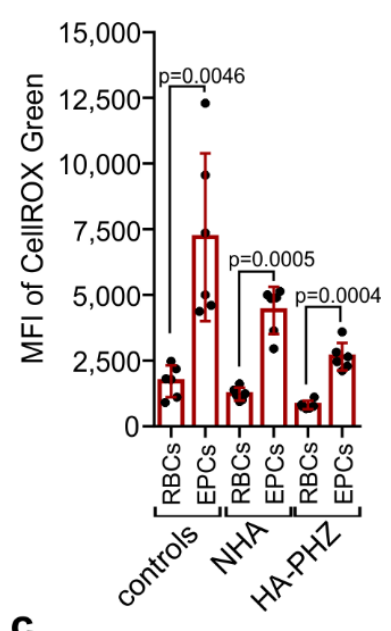

C

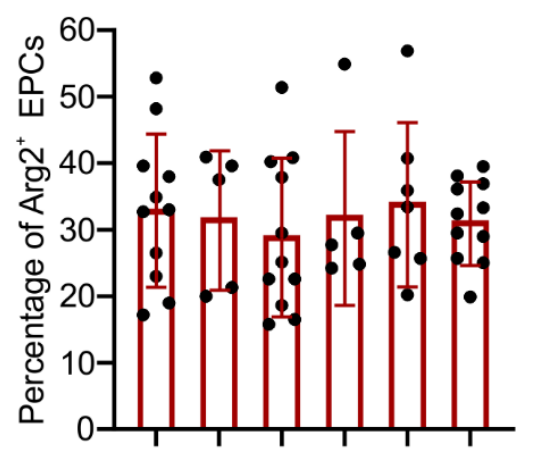

f

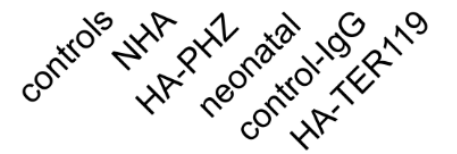

$\mathbf{f}$
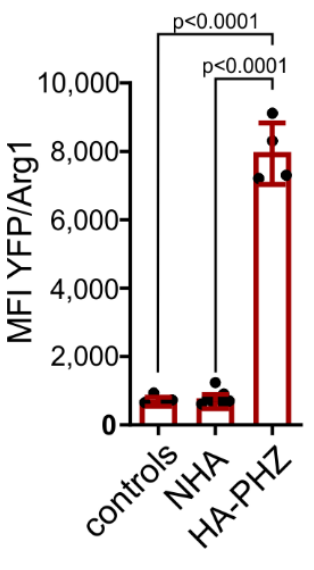

EPCs

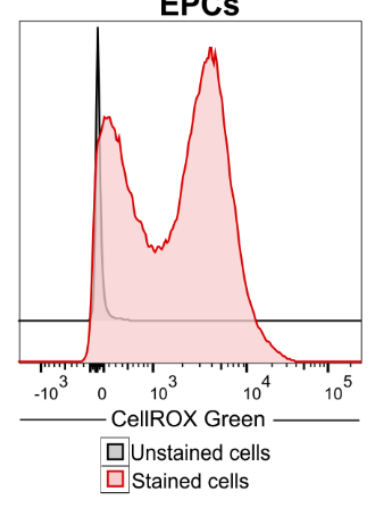

d

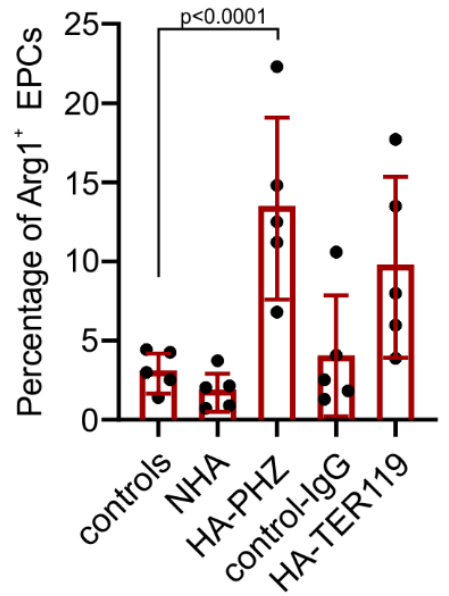

b

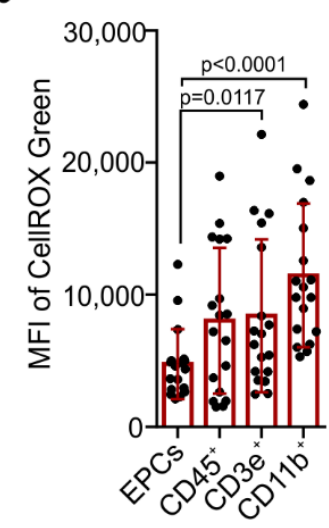

e

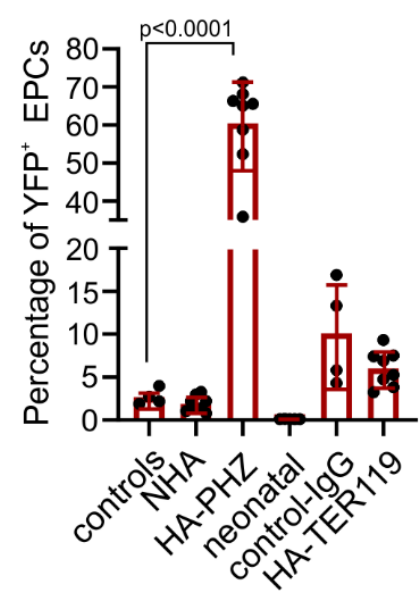

h

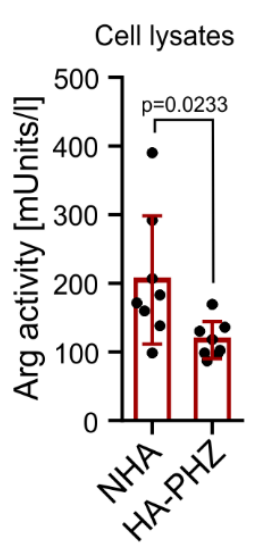

Supernatants

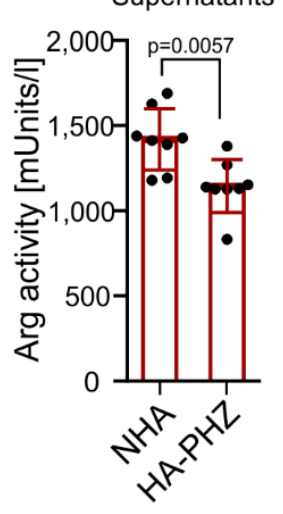


bioRxiv preprint doi: https://doi.org/10.1101/2021.01.18.427109; this version posted January 19, 2021. The copyright holder for this preprint (which was not certified by peer review) is the author/funder, who has granted bioRxiv a license to display the preprint in perpetuity. It is made available under aCC-BY-NC-ND 4.0 International license.

Figure 4.

a

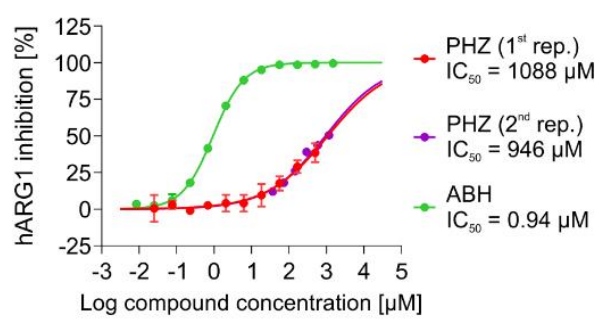

b

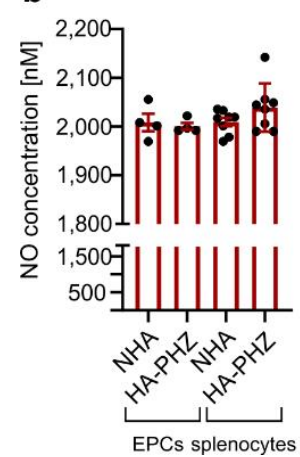

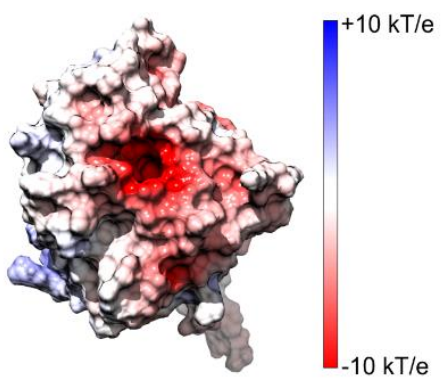

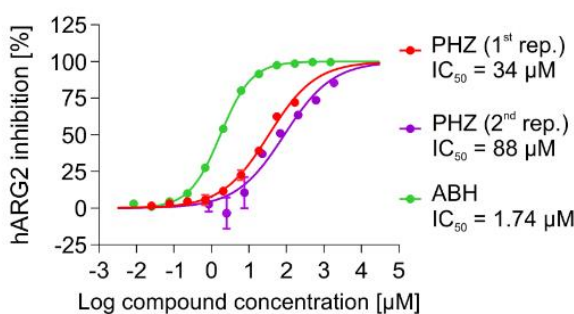

d

M. Neg $\mathrm{PHZ}+\mathrm{PHZ}$
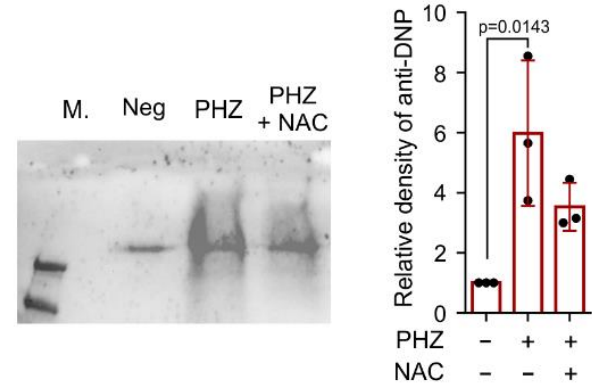

1096 
Figure 5.

a

C
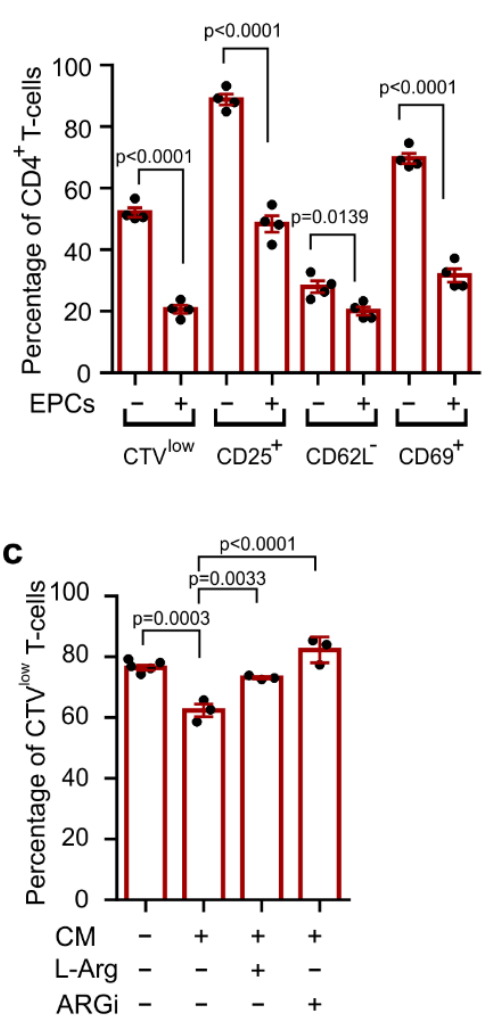
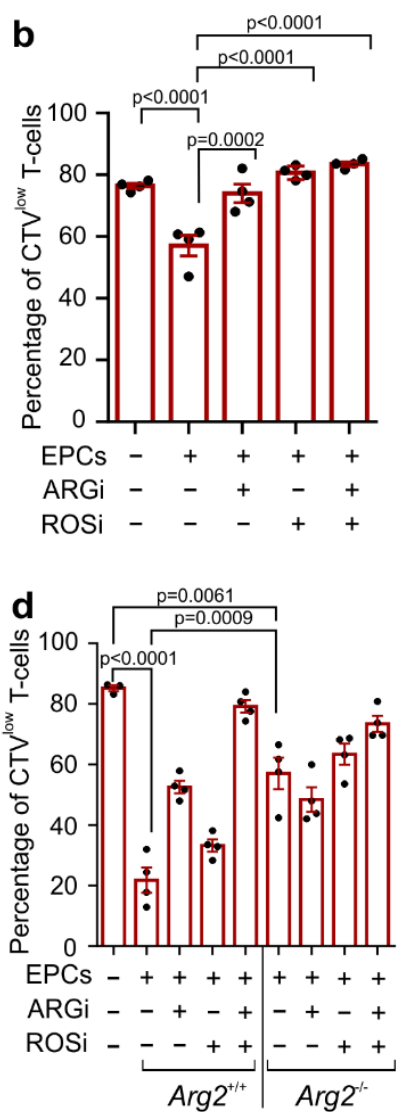
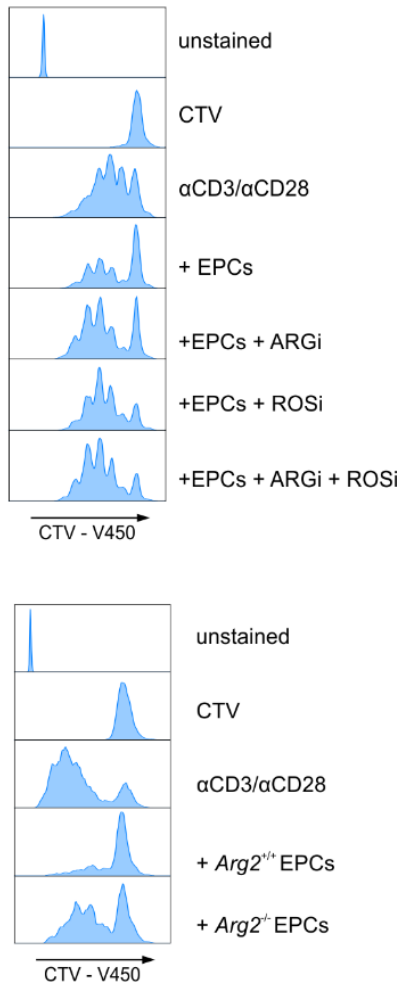

h
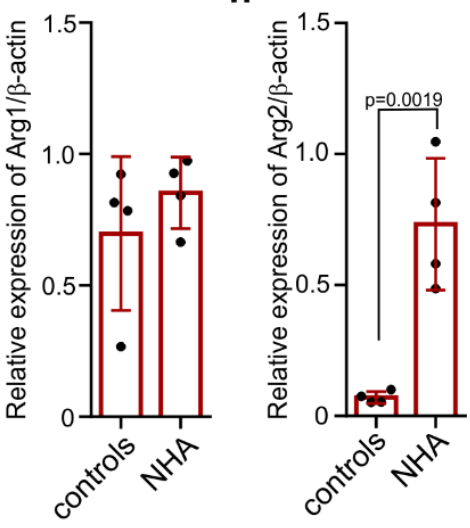

i

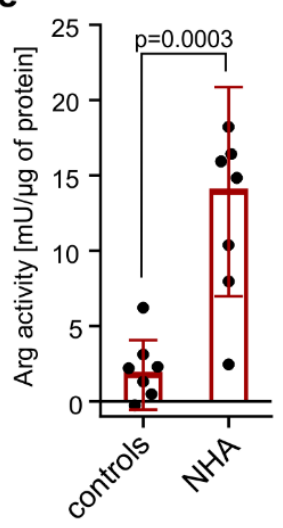

$\mathbf{f}$

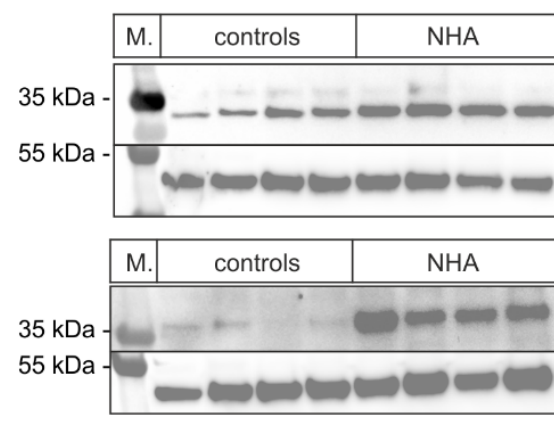

Arg2 $\beta$-actin

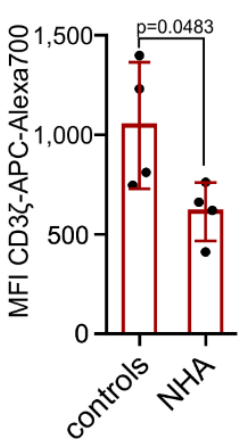

j

k

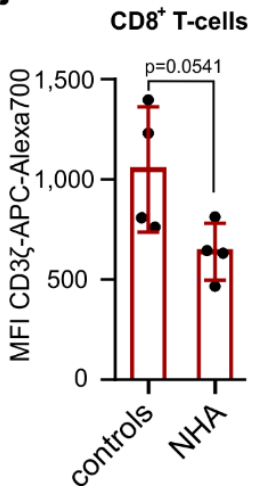

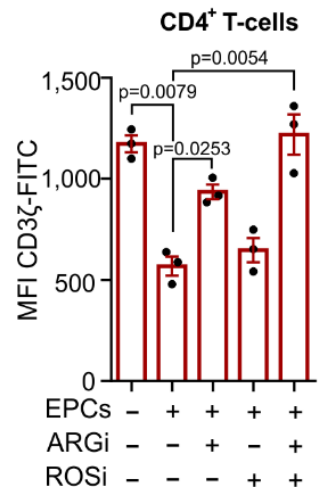

I

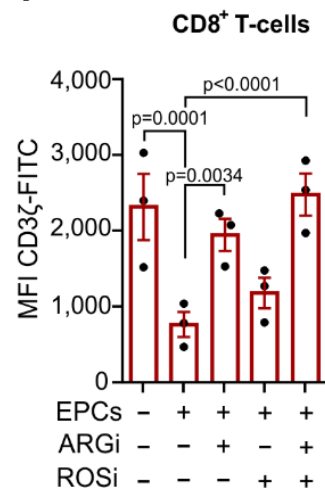


Figure 6.

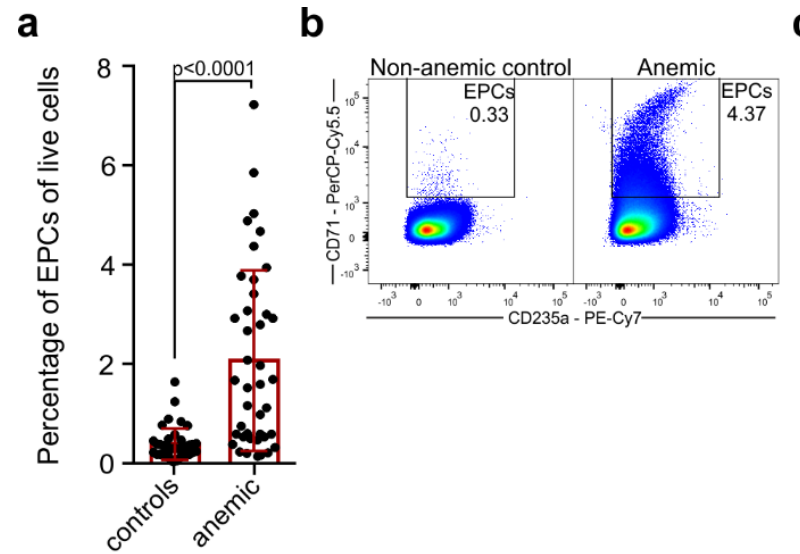

e

1102

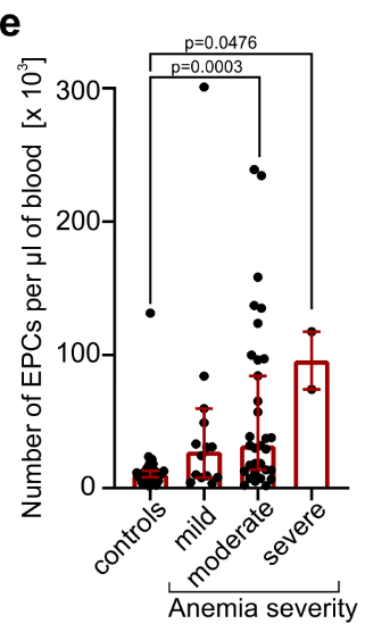

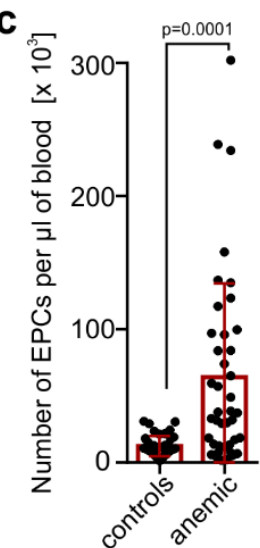

g
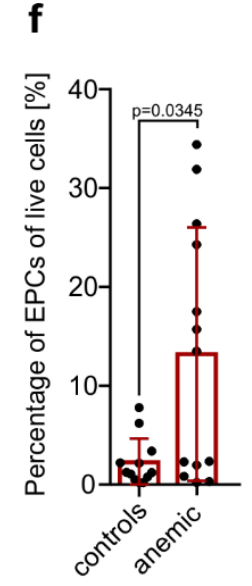

d

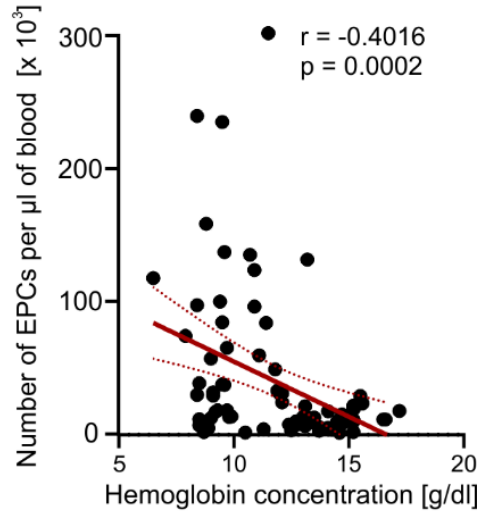

h

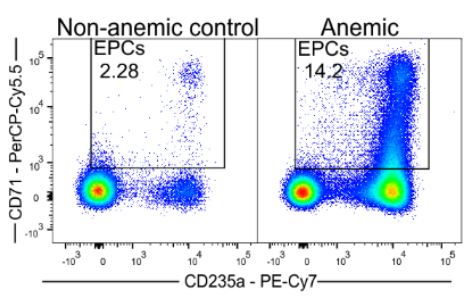

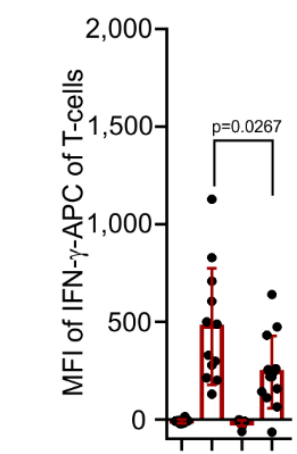

aCD3/aCD28 
a

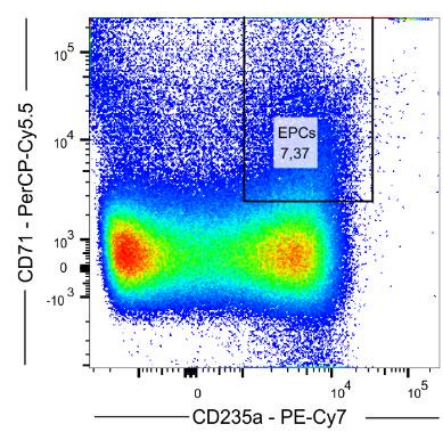

d

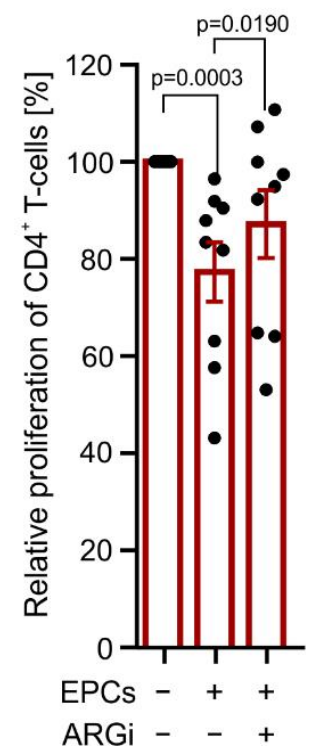

b

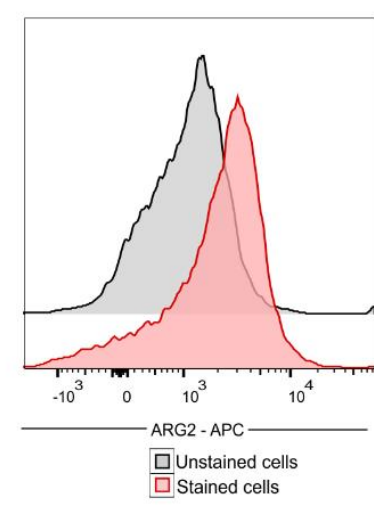

c

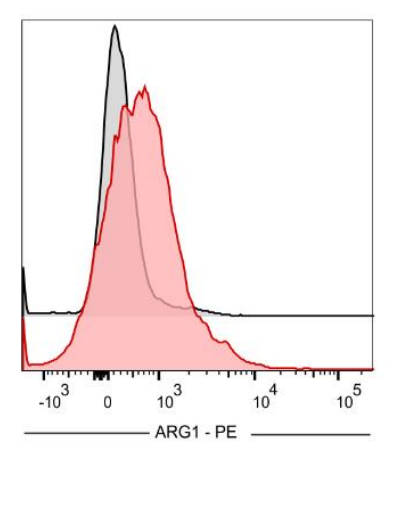

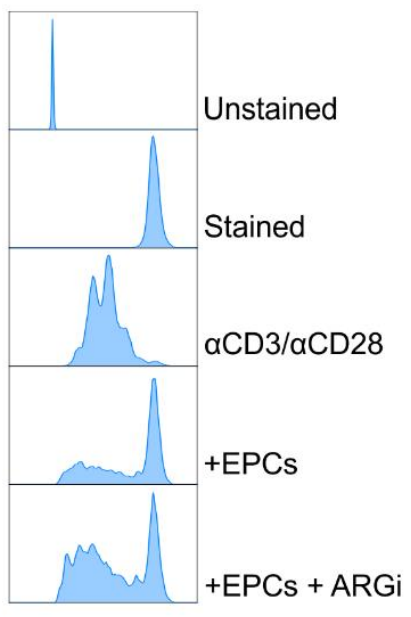
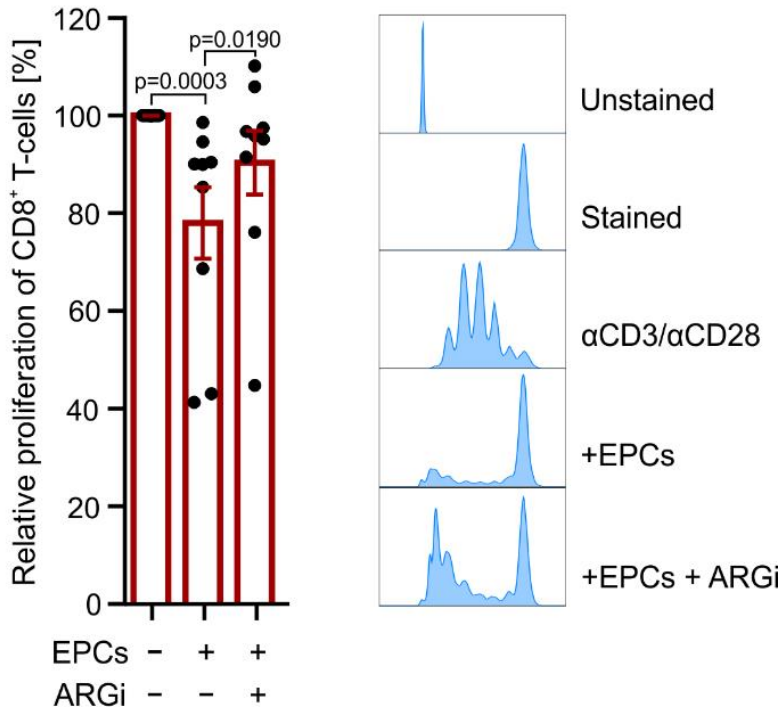
bioRxiv preprint doi: https://doi.org/10.1101/2021.01.18.427109; this version posted January 19,2021 . The copyright holder for this preprint (which was not certified by peer review) is the author/funder, who has granted bioRxiv a license to display the preprint in perpetuity. It is made available under aCC-BY-NC-ND 4.0 International license.

Figure 8.
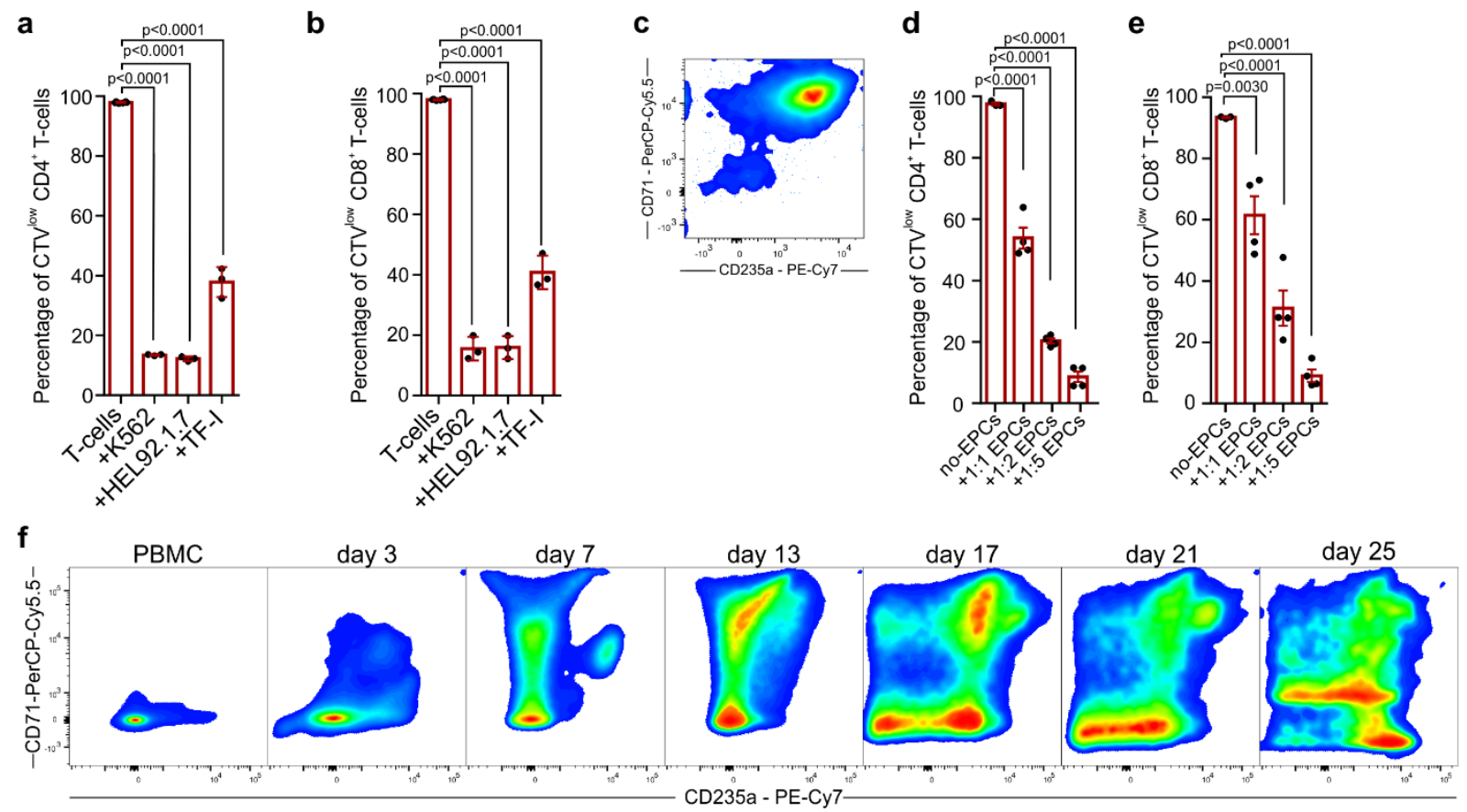

g
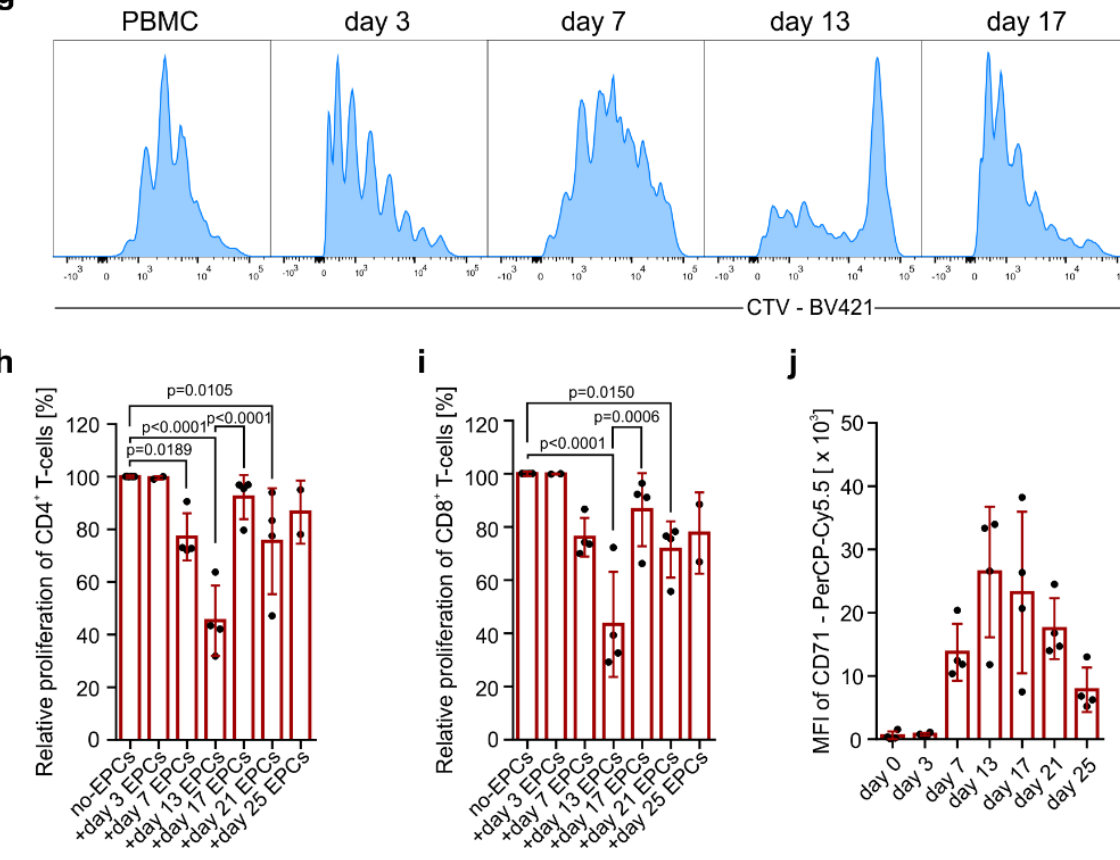

k

day 13

day 17

day 21

day 25

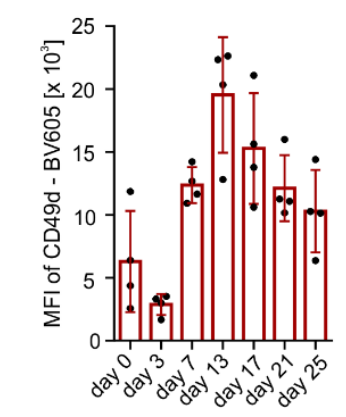

